\title{
PRODUCTS OF PAIRS OF DEHN TWISTS AND MAXIMAL REAL LEFSCHETZ FIBRATIONS
}

\author{
ALEX DEGTYAREV AND NERMİN SALEPCI
}

\begin{abstract}
We address the problem of existence and uniqueness of a factorization of a given element of the modular group into a product of two Dehn twists. As a geometric application, we conclude that any maximal real elliptic Lefschetz fibration is algebraic.
\end{abstract}

\section{$\S 1$. Introduction}

\subsection{Motivation}

An object repeatedly occurring in algebraic geometry is a fibration with singular fibers. If the base is a topological disk $D^{2}$ and the number of singular fibers is finite, the topology (and, in some extremal cases, the analytic structure as well) can adequately be described by the so-called monodromy factorization of the monodromy at infinity (the boundary of $D^{2}$ ).

More precisely, consider a proper smooth map $p: X \rightarrow B \cong D^{2}$, and let $\Delta:=\left\{b_{1}, b_{2}, \ldots, b_{r}\right\}$ be the set of the critical values of $p$, which are all assumed in the interior of $B$. The restriction of $p$ to $B^{\sharp}:=B \backslash \Delta$ is a locally trivial fibration, and one can consider its monodromy $\mathfrak{m}: \pi_{1}\left(B^{\sharp}, b\right) \rightarrow$ Aut $F_{b}$, where $F_{b}$ is the fiber over a fixed base point $b \in B^{\sharp}$ and where $G:=$ Aut $F_{b}$ is an appropriately defined group of classes of automorphisms of $F_{b}$. (The precise nature of the automorphisms used and their equivalence depend on a particular problem.) The monodromy at infinity $\mathfrak{m}_{\infty}:=\mathfrak{m}[\partial B] \in G$ is usually assumed fixed in advance.

WARNING. Throughout this paper, all group actions are right. (It is under this convention that monodromy is a homomorphism.) This convention applies to matrix groups as well: our matrices act on row vectors by the

Received October 21, 2011. Revised January 27, 2012. Accepted May 10, 2012.

2010 Mathematics Subject Classification. Primary 14P25, 57M60; Secondary 20F36, $11 \mathrm{~F} 06$.

Salepci's work was partially supported by the European Community's Seventh Framework Programme (FP7/2007-2013 and FP7/2007-2011) under grant agreement 258204 and by French National Research Agency grant ANR-08-BLAN-0291-02. 
right multiplication. Given a right action $X \times G \rightarrow X$, we denote by $x \uparrow g$ the image of $x \in X$ under $g \in G$.

Consider a system of lassos, one lasso $\gamma_{i}$ about each critical value $b_{i}$, $i=1, \ldots, r$, disjoint except at the common base point $b$ and such that $\gamma_{1} \ldots$. $\gamma_{r} \sim \partial B$. (Such a system is called a geometric basis for $\pi_{1}\left(B^{\sharp}, b\right)$.) Evaluating the monodromy $\mathfrak{m}$ at each $\gamma_{i}$, we obtain a sequence $\mathfrak{m}_{i}:=\mathfrak{m}\left(\gamma_{i}\right)$.

Definition 1.1. Given a group $G$, a $G$-valued monodromy factorization of length $r$ is a finite ordered sequence $\overline{\mathfrak{m}}:=\left(\mathfrak{m}_{1}, \ldots, \mathfrak{m}_{r}\right)$ of elements of $G$. The product $\mathfrak{m}_{\infty}:=\mathfrak{m}_{1} \cdot \ldots \cdot \mathfrak{m}_{r}$ is called the monodromy at infinity of $\overline{\mathfrak{m}}$, and $\overline{\mathfrak{m}}$ itself is often referred to as a monodromy factorization of $\mathfrak{m}_{\infty}$. The subgroup of $G$ generated by $\mathfrak{m}_{1}, \ldots, \mathfrak{m}_{r}$ is called the monodromy group of $\overline{\mathfrak{m}}$.

The ambiguity in the choice of a geometric basis leads to a certain equivalence relation. According to Artin [2], if $b \in \partial B$, any two geometric bases are related by an element of the braid group $\mathbb{B}_{r}$. Hence, the corresponding monodromy factorizations are related by a sequence of Hurwitz moves

$$
\sigma_{i}:\left(\ldots, \mathfrak{m}_{i}, \mathfrak{m}_{i+1}, \ldots\right) \mapsto\left(\ldots, \mathfrak{m}_{i} \mathfrak{m}_{i+1} \mathfrak{m}_{i}^{-1}, \mathfrak{m}_{i}, \ldots\right), \quad i=1, \ldots, r-1
$$

If the base point is not on the boundary or if the identification between $F_{b}$ and the "standard fiber" is not fixed, one should also consider the global conjugation

$$
g^{-1} \overline{\mathfrak{m}} g=\left(g^{-1} \mathfrak{m}_{1} g, \ldots, g^{-1} \mathfrak{m}_{r} g\right)
$$

by an element $g \in G$.

DEFINITION 1.3. Two monodromy factorizations are said to be strongly (resp., weakly) Hurwitz equivalent if they can be related by a finite sequence of Hurwitz moves (resp., a sequence of Hurwitz moves and global conjugation). For brevity, we routinely simplify this term to strong/weak equivalence.

It is immediate that both the monodromy at infinity and the monodromy group are invariant under strong Hurwitz equivalence, whereas their conjugacy classes are invariant under weak Hurwitz equivalence.

The best-known examples where this machinery applies are

- ramified coverings, with $G=\mathbb{S}_{n}$ the symmetric group;

- algebraic or, more generally, pseudoholomorphic curves in $\mathbb{C}^{2}$, with $G=$ $\mathbb{B}_{n}$ the braid group; 
- (real) elliptic surfaces or, more generally, (real) genus one Lefschetz fibrations, with $G=\tilde{\Gamma}:=\operatorname{SL}(2, \mathbb{Z})$ the mapping class group of a torus.

(Literature on the subject is abundant, and we direct the reader to [6] for further references.) Typically, the topological type of a singular fiber $F_{i}:=p^{-1}\left(b_{i}\right)$ is determined by the conjugacy class of the corresponding element $\mathfrak{m}_{i}$, and it is common to restrict the topological types by assuming that all $\mathfrak{m}_{i}$ should belong to a certain preselected set of conjugacy classes. Thus, in the three examples above, "simplest" singular fibers correspond to, respectively, transpositions in $\mathbb{S}_{n}$, Artin generators in $\mathbb{B}_{n}$, and Dehn twists in $\tilde{\Gamma}$ (see Section 2.1).

A monodromy factorization satisfying this additional restriction is often called simple, and a wide open problem with a great deal of possible geometric implications is the classification, up to strong/weak Hurwitz equivalence, of the simple monodromy factorizations of a given element $\mathfrak{m}_{\infty} \in G$ and of a given length.

\subsection{Principal results}

Geometrically, our principal subject is elliptic Lefschetz fibrations, and the algebraic counterpart is the classification of the factorizations of a given element $\mathfrak{m}_{\infty} \in \tilde{\Gamma}$ into products of Dehn twists. At this point, it is worth mentioning that there are cyclic central extensions $\tilde{\Gamma} \rightarrow \Gamma$ and $\mathbb{B}_{3} \rightarrow \Gamma$, where $\Gamma:=\operatorname{PSL}(2, \mathbb{Z})$ is the modular group, and each Dehn twist in $\Gamma$ lifts to a unique Dehn twist in $\tilde{\Gamma}$ or, respectively, to a unique Artin generator in $\mathbb{B}_{3}$; hence, the problems of the classification of simple monodromy factorizations in all three groups are equivalent. For this reason, we will mainly work in $\Gamma$. To simplify the further exposition, we introduce the following terminology: an $r$-factorization (of an element $g \in \Gamma$ ) is a monodromy factorization $\overline{\mathfrak{m}}=$ $\left(\mathfrak{m}_{1}, \ldots, \mathfrak{m}_{r}\right)$ with each $\mathfrak{m}_{i}$ a Dehn twist and such that $\mathfrak{m}_{\infty}=g$. To shorten the notation, we will often speak about an $r$-factorization $g=\mathfrak{m}_{1} \cdot \ldots \cdot \mathfrak{m}_{r}$.

Even with the group as simple as $\mathbb{B}_{3}$ (the first nonabelian braid group), surprisingly little is known. On the one hand, according to Moishezon and Livné (see [11]), a $6 k$-factorization of a power $\left(\sigma_{1} \sigma_{2}\right)^{3 k}$ of the Garside element is unique up to strong Hurwitz equivalence. This result was recently generalized by Orevkov [16] to any element positive in the standard Artin basis $\sigma_{1}, \sigma_{2}$. On the other hand, a series of exponentially large (in $r$ ) sets of 
nonequivalent $r$-factorizations of the same element $g_{r}:=L^{5 r-6} \in \Gamma$ (depending on $r$ ) was recently constructed in [6]; furthermore, these factorizations are indistinguishable by most conventional invariants. (For some other examples, related to the next braid group $\mathbb{B}_{4}$, see [8].)

Thus, it appears that, in its full generality, the problem of the classification of the $r$-factorizations of a given element is rather difficult and quite far from its complete understanding. In this paper, we confine ourselves to 2 -factorizations only, addressing both their existence and their uniqueness. Even in this simplest case, the results obtained seem rather unexpected.

Algebraically, our principal results are Theorems 1.4-1.6 below. For the statements, we briefly recall that the elements of the modular group are commonly divided into elliptic, parabolic, and hyperbolic, the former being those of finite order, and the two latter being those that, up to conjugation, can be represented by a word in positive powers of a particular pair $L, R$ of generators of $\Gamma$ (see Section 2.1 for further details). (Whenever speaking about words in a given alphabet, we mean positive words only; if negative powers are allowed, they are listed in the alphabet explicitly.) We use $A^{\mathrm{t}}$ for the transpose of a matrix $A$. One has $L^{\mathrm{t}}=R$; hence, the transpose $A^{\mathrm{t}}$ of a word $A$ in $\{L, R\}$ is again a word in $\{L, R\}$ : it is obtained from $A$ by interchanging $L \leftrightarrow R$ and reversing the order of the letters.

THEOREM 1.4. An element $g \in \Gamma$ admits a 2-factorization if and only if one of the following hold:

(1) $g \sim \mathbb{X}=R L^{-1}$ ( $g$ is elliptic),

(2) $g \sim R^{2}$ or $g \sim L^{4}$ (g is parabolic), or

(3) $g \sim L^{2} A L^{2} A^{\mathrm{t}}$ for some word $A \neq \varnothing$ in $\{L, R\}$ ( $g$ is hyperbolic).

THEOREM 1.5. The number of weak equivalence classes of 2-factorizations of $g \in \Gamma$ is at most one if $g$ is elliptic or parabolic, and at most two if $g$ is hyperbolic.

THEOREM 1.6. The single weak equivalence class of 2-factorizations of an element $g \sim L^{4}$ splits into two strong equivalence classes:

$$
L^{4}=R \cdot\left(R^{-1} L^{2}\right) R\left(R^{-1} L^{2}\right)^{-1}=L R L^{-1} \cdot\left(L R^{-1} L^{2}\right) R\left(L R^{-1} L^{2}\right)^{-1} .
$$

In all other cases, each weak equivalence class of 2-factorizations constitutes a single strong equivalence class.

Theorems 1.4, 1.5, and 1.6 are proved in Sections 3.1, 3.2, and 3.3, respectively. The proofs are based on a relation between subgroups of the modular 
group and a certain class of Grothendieck's dessins d'enfants. A refinement of Theorem 1.5, namely, a detailed description of the elements admitting more than one 2-factorization, is found in Section 3.4 (see Theorem 3.12).

Another interesting phenomenon related to the modular group is the fact that some of its elements are real — that is, they can be represented as a product of two involutive elements of $\operatorname{PGL}(2, \mathbb{Z}) \backslash \Gamma$. (For a geometric interpretation and further details, see [18] and Section 2.3.) The relation between this property and the existence/uniqueness of a 2-factorization, as well as the existence of real 2-factorizations, is discussed in Theorem 3.13.

Geometrically, 2-factorizations are related to real relatively minimal Jacobian elliptic Lefschetz fibrations over the sphere $S^{2}$ with two pairs of complex conjugate singular fibers; an important class of such fibrations comprises some maximal ones. Intuitively, an elliptic Lefschetz fibration is a topological counterpart of an algebraic elliptic surface (see Section 4 for the precise definitions), and one of the major questions is the realizability of a given real elliptic Lefschetz fibration by an algebraic one. (In the complex case, the answer to this question is trivially in the affirmative due to the classification found in [11] (see Theorem 4.1); in the real case, examples of nonalgebraic fibrations are known (see [20], [19]).) A real Lefschetz fibration is maximal if its real part has the maximal Betti number with respect to the Thom-Smith inequality (4.3). A maximal real Lefschetz fibration may have none, one, or two pairs of complex conjugate singular fibers (see Section 4.5). In the former case, the fibration is called totally real, and such a fibration is necessarily algebraic due to the following theorem.

Theorem 1.7 (see [20], [19]). Any totally real maximal Jacobian Lefschetz fibration is algebraic.

Among the most important geometric applications of the algebraic results of the paper is an extension of Theorem 1.7 to all maximal Jacobian fibrations.

THEOREM 1.8. Any maximal Jacobian Lefschetz fibration is algebraic.

This theorem is proved in Section 6.2.

As another geometric application, we settle a question left unanswered in [7]. Namely, we show that the equivariant deformation class of a nonsingular real trigonal $M$-curve in a Hirzebruch surface (see Section 5 for the definitions) is determined by the topology of its real structure (see Theorems 6.1 
and 6.3). Moreover, at most two such curves may share homeomorphic real parts.

One may speculate that it is the relation to maximal geometric objects, which are commonly known to be topologically "rigid," that makes 2-factorizations relatively "tame." At present, we have no clue what the general statements concerning the existence and uniqueness of $r$-factorizations may look like. One of the major reasons is that, even though an analogue of Proposition 2.8 holds for any number of Dehn twists, Lemma 3.2 does not have a literate extension to free groups on more than two generators (cf. [3]).

To our knowledge, even the finiteness of the number of equivalence classes of factorizations of a given element is still an open question. According to Matveyev and Rafi [10], certain finiteness statements do hold in hyperbolic groups; alas, neither $\Gamma$ nor $\mathbb{B}_{3}$ is hyperbolic. On the other hand, found in Moishezon [12] is an example of an infinite sequence of nonequivalent factorizations (although nonsimple) of the element $\Delta^{2}$ in the braid group $\mathbb{B}_{54}$.

\subsection{Contents of the paper}

Sections 2, 4, and 5 are of an auxiliary nature: we recall the basic notions and necessary known results concerning, respectively, the modular group, (real) elliptic Lefschetz fibrations, and (real) trigonal curves. The heart of the paper is Section 3: the principal algebraic results and their refinements are proved here. Section 6 deals with the geometric applications: we establish the semisimplicity of real trigonal $M$-curves and, as an upshot, prove Theorem 1.8 .

We use the conventional symbol $\square$ to mark the ends of the proofs. Some statements are marked with $\triangleleft$ or $\triangleright$ : the former means that the proof has already been explained (e.g., most corollaries), and the latter indicates that the proof is not found in the paper and the reader is directed to the literature, usually cited at the beginning of the statement.

\section{§. The modular group}

\subsection{Presentations of $\Gamma$}

Consider $\mathcal{H}=\mathbb{Z} \mathbf{a} \oplus \mathbb{Z} \mathbf{b}$, a rank 2 free abelian group with the skewsymmetric bilinear form $\bigwedge^{2} \mathcal{H} \rightarrow \mathbb{Z}$ given by $\mathbf{a} \cdot \mathbf{b}=1$. We regard $\tilde{\Gamma}:=$ $\mathrm{SL}(2, \mathbb{Z})$ as a group acting on $\mathcal{H}$. Moreover, $\tilde{\Gamma}$ is the group of symplectic 
autosymmetries of $\mathcal{H}$; it is generated by the matrices

$$
\mathbb{X}=\left[\begin{array}{cc}
1 & -1 \\
1 & 0
\end{array}\right], \quad \mathbb{Y}=\left[\begin{array}{cc}
0 & 1 \\
-1 & 0
\end{array}\right]
$$

such that $\mathbb{X}^{3}=-\mathrm{id}, \mathbb{Y}^{2}=-\mathrm{id}$.

The modular group $\Gamma:=\operatorname{PSL}(2, \mathbb{Z})$ is the quotient $\operatorname{SL}(2, \mathbb{Z}) / \pm \mathrm{id}$. When it does not lead to confusion, we use the same notation for a matrix $A$ in $\tilde{\Gamma}$ and its projection to $\Gamma$. It is known that $\Gamma \cong \mathbb{Z}_{3} * \mathbb{Z}_{2}$; we will work with the following two presentations of this group:

$$
\Gamma=\left\langle\mathbb{X}, \mathbb{Y}: \mathbb{X}^{3}=\mathbb{Y}^{2}=\mathrm{id}\right\rangle=\left\langle L, R: R L^{-1} R=L^{-1} R L^{-1},\left(R L^{-1}\right)^{3}=\mathrm{id}\right\rangle,
$$

where

$$
L=\left[\begin{array}{ll}
1 & 1 \\
0 & 1
\end{array}\right]=\mathbb{X} \mathbb{Y}, \quad R=\left[\begin{array}{ll}
1 & 0 \\
1 & 1
\end{array}\right]=\mathbb{X}^{2} \mathbb{Y},
$$

so that $\mathbb{X}=R L^{-1}$ and $\mathbb{Y}=L R^{-1} L=R^{-1} L R^{-1}$ in $\Gamma$. For future reference, note that the powers of these matrices are given by

$$
L^{n}=\left[\begin{array}{ll}
1 & n \\
0 & 1
\end{array}\right], \quad R^{n}=\left[\begin{array}{ll}
1 & 0 \\
n & 1
\end{array}\right], \quad n \in \mathbb{Z} .
$$

Since $\Gamma$ is a free product of cyclic groups, we have the following statement.

Lemma 2.1. Two elements $f, g \in \Gamma$ commute if and only if they generate a cyclic subgroup or, equivalently, if they are both powers of a common element $h \in \Gamma$.

\subsection{The conjugacy classes}

A simple way to understand the conjugacy classes is via the action of $\Gamma$ on the Poincaré disk. The group $\Gamma$ is known to be the symmetry group of the Poincaré disk endowed with the so-called Farey tessellation, shown in Figure 1. The nontrivial elements of $\Gamma$ form three basic families: elliptic, parabolic, and hyperbolic. These families are distinguished by the nature of their fixed points on the Poincaré disk or, equivalently, by the absolute value of their traces. An elliptic matrix has $\mid$ trace $\mid<2$, so that it has a single fixed point in the interior of the Poincaré disk and acts as a rotation with respect to this fixed point. Elliptic matrices are the only torsion elements of $\Gamma$. A parabolic matrix has $\mid$ trace $\mid=2$; it has a single rational fixed point (on the boundary of the Poincaré disk) and acts as a rotation fixing 


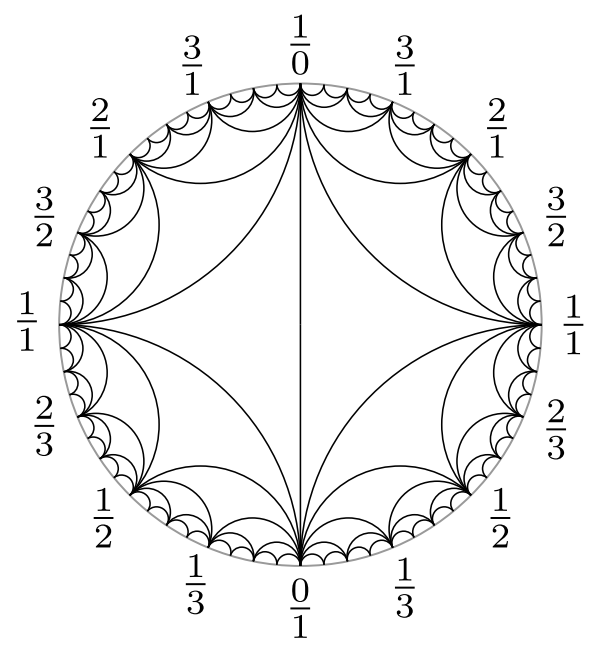

Figure 1: Poincaré disk endowed with the Farey tessellation.

this boundary point. A hyperbolic matrix, defined via $\mid$ trace $\mid>2$, has two irrational fixed points on the boundary and acts as a translation fixing the geodesic connecting these fixed points.

There are three conjugacy classes of elliptic matrices. Representatives of these classes can be taken as

$$
\mathbb{Y}=\left[\begin{array}{cc}
0 & 1 \\
-1 & 0
\end{array}\right], \quad \mathbb{X}=\left[\begin{array}{cc}
1 & -1 \\
1 & 0
\end{array}\right], \quad \mathbb{X}^{-1}=\left[\begin{array}{cc}
0 & 1 \\
-1 & 1
\end{array}\right]
$$

An element in $\Gamma$ is called a (positive) Dehn twist if it is conjugate to $R$. (The geometric meaning of this definition is explained in Section 2.4.) Any parabolic element is conjugate to a certain $n$th power of a Dehn twist. Thus, a representative of a class can be taken as $R^{n}$.

WARning. For the experts, we emphasize that, in accordance with our right-group action convention, it is $R$, not $L$, that represents a positive Dehn twist.

The conjugacy classes of hyperbolic elements of $\Gamma$ are determined by sequences $\left[a_{1}, a_{2}, \ldots, a_{2 n}\right], a_{i} \in \mathbb{Z}^{+}$, defined up to even permutations and called cutting period cycles. Indeed, the fixed points of a hyperbolic matrix are irrational points that are the zeros of a quadratic equation, and they 
have a continued fraction expansion with the periodic tail

$$
\cdots a_{1}+\frac{1}{a_{2}+\frac{1}{\cdot \cdot \frac{1}{a_{2 n}}}}
$$

Note that $\left[a_{1}, a_{2}, \ldots, a_{2 n}\right]$ is not necessarily the minimal period: all matrices sharing the same pair of eigenvectors are powers of a minimal one, and the precise multiple of the minimal period corresponding to a given matrix $A$ can be recovered from its trace.

A representative of the conjugacy class corresponding to a cutting period cycle $\left[a_{1}, a_{2}, \ldots, a_{2 n}\right]$ can be chosen as

$$
R^{a_{1}} \cdot L^{a_{2}} \cdot \ldots \cdot L^{a_{2 n}}=\left[\begin{array}{cc}
1 & 0 \\
a_{1} & 1
\end{array}\right] \cdot\left[\begin{array}{cc}
1 & a_{2} \\
0 & 1
\end{array}\right] \cdot \ldots \cdot\left[\begin{array}{cc}
1 & a_{n} \\
0 & 1
\end{array}\right]
$$

We will be interested not only in the cutting period cycle but also in the underlying word, called the cutting word, in two letters $\{L, R\}$. Recall that the cutting word encodes the two types (right/left) of triangles of the Farey tessellation cut by the invariant geodesic (cf. [18], [21]). In terms of the cutting word, hyperbolic conjugacy classes can be characterized as those represented by a word in $\{L, R\}$ with both $L$ and $R$ present. Since the cutting word is defined only up to cyclic permutation, it is convenient to represent it in the unit circle, placing the letters constituting the word at equal angles (cf. Figure 6). The resulting circle marked with a number of copies of $L$ and $R$ is called the cyclic diagram $\mathcal{D}_{g}$ of a hyperbolic element $g$. One can also speak about the cyclic diagram of a parabolic element, with the letters either all $R$ (for a positive power of a Dehn twist) or all $L$ (for a negative power).

\subsection{Real elements}

An involutive element of the coset $\operatorname{PGL}(2, \mathbb{Z}) \backslash \Gamma$ is called a real structure on $\Gamma$. An element of $\Gamma$ is called real if, in $\operatorname{PGL}(2, \mathbb{Z})$, it has a decomposition into a product of two real structures. For any real structure $\tau$, let us define an involutive antiautomorphism $\hat{\tau}: \Gamma \rightarrow \Gamma$ given by $\hat{\tau}(g)=\tau g^{-1} \tau$. Then, a real element can also be defined as one fixed by $\hat{\tau}$ for some real structure $\tau$. The significance of real elements is in their geometric interpretation. For example, such an element appears as the $\Gamma$-valued monodromy at infinity of a real elliptic Lefschetz fibration over a disk. 
The characterization of real elements in $\Gamma$, as well as in $\tilde{\Gamma}$, is known (see [18]): all elliptic and parabolic matrices are real, and a hyperbolic matrix is real if and only if its cutting period cycle is odd bipalindromic - that is, up to cyclic permutation, it is a union of two palindromic pieces of odd length. This property can be interpreted in terms of the cyclic diagram as the existence of a symmetry axis such that the diagram is invariant under the reflection with respect to this axis.

Up to conjugation, there are exactly two real structures on $\Gamma$ :

$$
\tau_{1}=\left[\begin{array}{ll}
0 & 1 \\
1 & 0
\end{array}\right], \quad \tau_{2}=\left[\begin{array}{cc}
1 & 0 \\
0 & -1
\end{array}\right] .
$$

In the rest of the paper, $\tau_{1}$ and $\tau_{2}$ refer to these particular matrices. The action of $\hat{\tau}_{i}$ on the generators is as follows:

$$
\begin{array}{llll}
\hat{\tau}_{1}(L)=R^{-1}, & \hat{\tau}_{1}(R)=L^{-1}, & \hat{\tau}_{1}(\mathbb{X})=\mathbb{X}, & \hat{\tau}_{1}(\mathbb{Y})=\mathbb{Y}, \\
\hat{\tau}_{2}(L)=L, & \hat{\tau}_{2}(R)=R, & \hat{\tau}_{2}(\mathbb{X})=\mathbb{Y} \mathbb{X}, & \hat{\tau}_{2}(\mathbb{Y})=\mathbb{Y} .
\end{array}
$$

We extend the antiautomorphism $\hat{\tau}: \Gamma \rightarrow \Gamma$ to the set of $\Gamma$-valued monodromy factorizations as follows:

$$
\hat{\tau}\left(\mathfrak{m}_{1}, \ldots, \mathfrak{m}_{r}\right)=\left(\hat{\tau}\left(\mathfrak{m}_{r}\right), \ldots, \hat{\tau}\left(\mathfrak{m}_{1}\right)\right) .
$$

(Note the reverse order.) It is straightforward that the factorizations $\hat{\tau}\left(\overline{\mathfrak{m}}^{\prime}\right)$ and $\hat{\tau}\left(\overline{\mathfrak{m}}^{\prime \prime}\right)$ are strongly/weakly equivalent if and only if $\overline{\mathfrak{m}}^{\prime}$ and $\overline{\mathfrak{m}}^{\prime \prime}$ are. Furthermore, one has $\hat{\tau}(\overline{\mathfrak{m}})_{\infty}=\hat{\tau}\left(\mathfrak{m}_{\infty}\right)$, and the monodromy group of $\hat{\tau}(\overline{\mathfrak{m}})$ is the image of that of $\overline{\mathfrak{m}}$ under $\hat{\tau}$.

With future applications in mind, we will also discuss real 2-factorizations. A 2 -factorization $\overline{\mathfrak{m}}$ is said to be real if there is a real structure $\tau$ such that either $\hat{\tau}(\overline{\mathfrak{m}})=\overline{\mathfrak{m}} \uparrow \sigma_{1}$ (see $\left.(1.2)\right)$ or $\hat{\tau}(\overline{\mathfrak{m}})=\overline{\mathfrak{m}}$. The monodromy at infinity of a real 2-factorization is obviously real; the converse is not true (see [17], [18] and Section 3.5).

REMARK 2.5. Geometrically, a real 2-factorization represents a real Jacobian Lefschetz fibration over the unit disk $D^{2} \subset \mathbb{C}$ (with the standard real structure $z \mapsto \bar{z}$ ) with two singular fibers (see Section 4.2); in the former case $\left(\hat{\tau}(\overline{\mathfrak{m}})=\overline{\mathfrak{m}} \uparrow \sigma_{1}\right)$, the two singular fibers are real; in the latter case $(\hat{\tau}(\overline{\mathfrak{m}})=\overline{\mathfrak{m}})$, they are complex conjugate. A specific example of a nonreal 2-factorization with real monodromy at infinity is studied in [17]; this example has interesting geometric implications. 
REMARK 2.6. Alternatively, a 2-factorization $\overline{\mathfrak{m}}$ is real if and only if $\hat{\tau}(\overline{\mathfrak{m}})$ is strongly Hurwitz equivalent to $\overline{\mathfrak{m}}$ for some real structure $\tau$. Indeed, since an even power $\sigma_{1}^{2 k}$ acts via the conjugation by the $\tau$-real element $\mathfrak{m}_{\infty}^{-k}$, it can be "undone" by replacing $\tau$ with $\tau^{\prime}:=\tau \mathfrak{m}_{\infty}^{k}$, which is also a real structure. In particular, it follows that being real is a property of a whole strong Hurwitz equivalence class.

\subsection{The mapping class group}

The mapping class group $\operatorname{Map}_{+}(S)$ of an oriented smooth surface $S$ is defined as the group of isotopy classes of orientation-preserving diffeomorphisms of $S$. If $S$ is the 2-torus $T^{2}$, one can fix an isomorphism $H_{1}\left(T^{2}\right.$, $\mathbb{Z}) \cong \mathcal{H}=\mathbb{Z} \mathbf{a} \oplus \mathbb{Z} \mathbf{b}$, and the map $f \mapsto f_{*}$ establishes an isomorphism $\operatorname{Map}_{+}\left(T^{2}\right) \rightarrow \tilde{\Gamma}$.

The (positive) Dehn twist along a simple closed curve $l \in S$ is a diffeomorphism of $S$ obtained by cutting $S$ along $l$ and regluing with a twist of $2 \pi$. If $S \cong T^{2}$, the image of the Dehn twist in the mapping class group $\tilde{\Gamma}$ depends only on the homology class $u:=[l] \in \mathcal{H}$ and is given by the symplectic reflection $x \mapsto x+(u, x) u$, where $(u, x)$ denotes the algebraic sum of the points of intersection of $u$ an $x$; we denote this image by $t_{u}$ and call it a Dehn twist in $\tilde{\Gamma}$. All Dehn twists form a whole conjugacy class which contains $R$; they project to the positive Dehn twists in $\Gamma$ introduced in Section 2.2.

\subsection{Subgroups of $\Gamma$}

In this section, we summarize the relation between the subgroups of $\Gamma$ and a special class of bipartite ribbon graphs, which we call skeletons. A similar approach, in terms of special triangulations of surfaces, was developed in [4]. Our approach is identical to the bipartite cuboid graphs in [9], except that we are mainly interested in subgroups of infinite index and therefore are forced to consider infinite graphs supported by noncompact surfaces. We only recall briefly the few definitions and facts needed later in this paper; for details and further references, see [6]. Note that, due to our right-group action convention, some definitions given below differ slightly from those in $[6]$.

Recall that a ribbon graph is a graph (locally finite $C W$-complex of dimension 1), possibly infinite, equipped with a cyclic order (i.e., transitive $\mathbb{Z}$-action) on the star of each vertex. Typically, a ribbon graph is a graph embedded into an oriented surface $S$, and the cyclic order is induced by the orientation of $S$. In fact, a ribbon graph $\mathfrak{G}$ defines a unique, up to homeomorphism, minimal oriented surface $S_{0}$ (noncompact if $\mathfrak{G}$ is infinite) 
into which it is embedded. The connected components of the complement $S_{0} \backslash \mathfrak{G}$ are called the regions of $\mathfrak{G}$.

A bipartite graph is a graph whose vertices are colored with two colors, - and $\circ$, so that each edge connects vertices of opposite colors.

Definition 2.7. A skeleton is a connected bipartite ribbon graph with all •-vertices of valency 3 or 1 and all o-vertices of valency 2 or 1 . A skeleton is regular if all its •- and o-vertices have valency 3 and 2, respectively.

Since $\Gamma=\left\{\mathbb{X}, \mathbb{Y}: \mathbb{X}^{3}=\mathbb{Y}^{2}=\mathrm{id}\right\}$, the set of edges of any skeleton is a transitive $\Gamma$-set, with the action of $\mathbb{X}$ and $\mathbb{Y}$ given by the distinguished cyclic order on the stars of, respectively, •- and o-vertices. (Because of the valency restrictions in Definition 5.6 below, this action of $\mathbb{Z} * \mathbb{Z}$ does factor through $\Gamma$.) Conversely, any transitive $\Gamma$-set can be regarded as (the set of edges of) a skeleton, the $\bullet$ - and o-vertices being the orbits of $\mathbb{X}$ and $\mathbb{Y}$, respectively. We identify the two categories.

As a consequence, to each subgroup $G \subset \Gamma$ one can associate the skeleton $G \backslash \Gamma$ (the set of left $G$-cosets, regarded as a right $\Gamma$-set). This skeleton is regular if and only if $G$ is torsion-free, that is, contains no elliptic elements; in this case, $G$ is free. The skeleton $G \backslash \Gamma$ is equipped with a distinguished edge $e:=G \backslash G$, which we call the base point. Conversely, given a skeleton $\mathfrak{S}$ and a base point $e$, the stabilizer $G$ of $e$ is a subgroup of $\Gamma$, and one has $\mathfrak{S}=G \backslash \Gamma$. In general, without a base point chosen, the stabilizer of $\mathfrak{S}$ is defined as a conjugacy class of subgroups of $\Gamma$.

Convention. In the figures, we usually omit most bivalent o-vertices, assuming that such a vertex is to be inserted at the center of each "edge" connecting a pair of $\bullet$-vertices. When of interest, the base point is denoted by a gray diamond. For infinite skeletons, only a compact part is drawn, and each maximal Farey branch (see Section 2.6 and Figure 2, left, below) is represented by a $\Delta$-vertex.

A combinatorial path (called a chain in [6]) in a skeleton $\mathfrak{S}$ can be regarded as a pair $\gamma:=\left(e^{\prime}, g\right)$, where $e^{\prime}$ is an edge, called the initial point of $\gamma$, and $g \in \Gamma$. Then $e^{\prime \prime}:=e^{\prime} \uparrow g$ is the terminal point of $\gamma$, and the evaluation map val $: \gamma \mapsto g$ sends a path $\gamma=\left(e^{\prime}, g\right)$ to its underlying element $g \in \Gamma$. For a regular skeleton $\mathfrak{S}$, the map val establishes an isomorphism $\pi_{1}(\mathfrak{S}, e)=G$. (In the presence of monovalent vertices, one should replace $\pi_{1}$ with an appropriate orbifold fundamental group.) When the initial point is understood, we identify a path $\gamma$ and its image val $\gamma \in \Gamma$. The product 
of two paths is defined as usual: $\left(e^{\prime}, g^{\prime}\right) \cdot\left(e^{\prime \prime}, g^{\prime \prime}\right)=\left(e^{\prime}, g^{\prime} g^{\prime \prime}\right)$, provided that $e^{\prime \prime}=e^{\prime} \uparrow g^{\prime}$; the inverse of $\gamma=\left(e^{\prime}, g\right)$ is $\gamma^{-1}:=\left(e^{\prime} \uparrow g, g^{-1}\right)$.

In the case of skeletons, a region can be redefined as an orbit of $L=\mathbb{X} \mathbb{Y}$. In this definition, a region $\mathcal{R}$ is the set of edges in the boundary of the geometric realization of $\mathcal{R}$ whose canonical orientation $\bullet \rightarrow 0$ agrees with the boundary orientation; the other edges in the boundary are of the form $e \uparrow \mathbb{Y}, e \in \mathcal{R}$. An $n$-gonal region is an orbit of length $n$; intuitively, $n$ is the number of $\bullet$-vertices in the boundary. The minimal supporting surface $S_{0}$ of a skeleton $\mathfrak{S}$ can be obtained by patching the boundary of each region $\mathcal{R}$ with a disk (if $\mathcal{R}$ is finite) or half-plane (if $\mathcal{R}$ is infinite).

Given a subgroup $G \subset \Gamma$, the $G$-conjugacy classes of the Dehn twist contained in $G$ are in a canonical one-to-one correspondence with the monogonal regions of the skeleton $G \backslash \Gamma$ (see [6]): under the canonical identification $G=\pi_{1}^{\text {orb }}(G \backslash \Gamma, G \backslash G)$ described above, these classes are realized by the boundaries of the monogons.

\subsection{Pseudotrees}

A special class of skeletons can be obtained from ribbon trees as follows. Consider a ribbon tree with all •-vertices of valency 3 (nodes) or 1 (leaves), and take its bipartite subdivision - that is, divide each edge into two by inserting an extra o-vertex in the middle. We denote the resulting graph by $\mathfrak{G}$. Let us consider a vertex function $\ell:\{$ leaves $\} \rightarrow\{0, \Delta, \bullet, \circ\}$ such that, if two leaves are incident to a common node, then $\ell$ does not assign $\Delta$ to both. We perform the following modifications at each leaf $v$ of $\mathfrak{G}$ :

- if $\ell(v)=\bullet$, then no modification is done;

- if $\ell(v)=\circ$, then cut out the leaf and the incident edge, so that the resulting graph has a monovalent o-vertex;

- if $\ell(v)=0$, then splice $\mathfrak{G}$ with a simple loop (see Figure 2, left);

- if $\ell(v)=\Delta$, then splice $\mathfrak{G}$ with a Farey branch (see Figure 2, right).
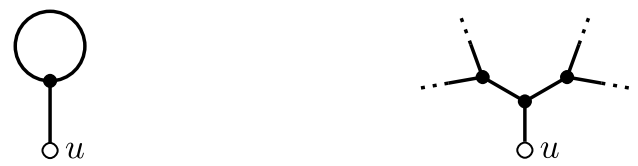

Figure 2: A simple loop and a Farey branch. 


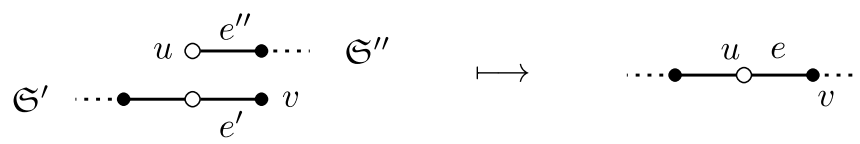

Figure 3: The splice of two skeletons.

Formally, a simple loop is the skeleton $\Gamma_{1}(2) \backslash \Gamma$, where $\Gamma_{1}(2)=$ $\left\{\left[\begin{array}{ll}1 & 0 \\ * & 1\end{array}\right] \bmod 2\right\}$, and a Farey branch $\mathbb{Y} \backslash \Gamma$ is the only bipartite ribbon tree regular except a single monovalent vertex, which is ०. Given two skeletons $\mathfrak{S}^{\prime}, \mathfrak{S}^{\prime \prime}$, a monovalent •-vertex $v$ of $\mathfrak{S}^{\prime}$, and a monovalent o-vertex $u$ of $\mathfrak{S}^{\prime \prime}$, the splice is defined as the skeleton obtained from the disjoint union $\mathfrak{S}^{\prime} \sqcup \mathfrak{S}^{\prime \prime}$ by identifying the edges $e^{\prime}, e^{\prime \prime}$ incident to $v, u$, respectively, to a common edge $e$ (see Figure 3).

A skeleton that can be obtained by the above procedure is called a pseudotree. A pseudotree is regular if and only if the images of $\ell$ are in $\{0, \Delta\}$.

Crucial for the proofs is the following statement, which is an immediate consequence of [6, Proposition 4.4].

Proposition 2.8. A proper subgroup $G \subset \Gamma$ is generated by two distinct Dehn twists if and only if its skeleton $\mathfrak{S}:=G \backslash \Gamma$ is a regular pseudotree with exactly two simple loops. In this case, $G$ is freely generated by two Dehn twists.

Because of the requirement on the $\Delta$-values of a vertex function, a pseudotree $\mathfrak{S}$ as in Proposition 2.8 looks as shown in Figure 4. More precisely, $\mathfrak{S}$ consists of two monogons connected by a horizontal line segment and a number of Farey branches, upward and downward, attached to this segment. Thus, starting from one of the monogons, one can encode $\mathfrak{S}$ and, hence, the subgroup $G$ itself by the sequence of the directions (up/down) of the Farey branches.

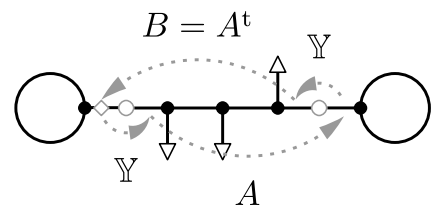

Figure 4: An example of a pseudotree. 
REMARK 2.9. The monodromy at infinity of a pseudotree $\mathfrak{S}$ is the conjugacy class $\mathfrak{m}_{\infty}$ in $\Gamma$ realized by a large circle encompassing the compact part of $\mathfrak{S}$. Let us choose the base point $e$ next to one of the monogons as shown in Figure 4. Starting from $e$, we can realize $\mathfrak{m}_{\infty}$ by the element $L^{2} A L^{2} B$, where $A$ and $B$ are the paths shown in the figure. Namely, $A$ starts at $e^{\prime}:=e \uparrow(\mathbb{X} \mathbb{Y})^{2}=e \uparrow L^{2}=e \uparrow \mathbb{Y}$ and is a product of copies of $R=\mathbb{X}^{2} \mathbb{Y}$ and $L=\mathbb{X} \mathbb{Y}$, each downward $\Delta$-vertex contributing an $R$ and each upward $\Delta$ vertex contributing an $L$. The other path $B$ can be described similarly starting from a base point next to the other monogonal region. However, it is obvious from the figure that the loop $(e, \mathbb{Y} A \mathbb{Y} B)$ is contractible. Hence, $B=\mathbb{Y} A^{-1} \mathbb{Y}$, and one can easily verify that $A^{\mathrm{t}}=\mathbb{Y} A^{-1} \mathbb{Y}$ in $\Gamma$. Thus, we arrive at

$$
\mathfrak{m}_{\infty} \sim L^{2} A L^{2} A^{\mathrm{t}}
$$

where the word $A$ in $\{L, R\}$ (possibly empty) is as described above. As an upshot of this description, we have the converse statement: a representation of the monodromy at infinity in the form (2.10) determines a pseudotree up to isomorphism.

\section{$\S 3 . \quad$ The classification of 2-factorizations}

\subsection{Proof of Theorem $\mathbf{1 . 4}$}

We precede the proof of this theorem with a few auxiliary statements.

Lemma 3.1. Two Dehn twists $t_{u}, t_{v}, u, v \in \mathcal{H}$, generate $\Gamma$ if and only if $u$ and $v$ span $\mathcal{H}$. If this is the case, the pair $\left(t_{u}, t_{v}\right)$ is conjugate to $\left(R, L^{-1}\right)$.

Proof. If $u$ and $v$ span $\mathcal{H}$, the signs can be chosen so that the matrix $M$ formed by $u, v$ as rows has determinant 1 , that is, belongs to $\tilde{\Gamma}$. The conjugation by $M$ takes $\left(R, L^{-1}\right)$ to $\left(t_{u}, t_{v}\right)$; hence, $t_{u}$ and $t_{v}$ generate $\Gamma$.

For the converse statement, assume that the subgroup $\mathcal{H}^{\prime} \subset \mathcal{H}$ spanned by $u$ and $v$ is proper. Since Dehn twists are symplectic reflections (see Section 2.4), the subgroup $\mathcal{H}^{\prime}$ is obviously invariant under the subgroup $\Gamma^{\prime} \subset \Gamma$ generated by $t_{u}$ and $t_{v}$. Thus, there are primitive vectors in $\mathcal{H}$ that are not in the orbit $u \uparrow \Gamma^{\prime}$. On the other hand, all primitive vectors are known to form a single $\Gamma$-orbit; hence, $\Gamma^{\prime} \subset \Gamma$ is a proper subgroup.

Lemma 3.2 (cf. Bardakov [3]). Let $G:=\langle\alpha, \beta\rangle$ be a free group, and let $\alpha^{\prime}, \beta^{\prime} \in G$ be two elements generating $G$ and such that each $\alpha^{\prime}, \beta^{\prime}$ is conjugate to one of the original generators $\alpha, \beta$. Then the pair $\left(\alpha^{\prime}, \beta^{\prime}\right)$ is weakly Hurwitz equivalent to $(\alpha, \beta)$. 
Proof. After a global conjugation, possibly followed by $\sigma_{1}$, one can assume that $\alpha^{\prime}=\alpha$. Then obviously $\beta^{\prime}=T^{-1} \beta T$ for some reduced word $T$ in $\left\{\alpha^{ \pm 1}, \beta^{ \pm 1}\right\}$. One can assume that the first letter of $T$ is not $\beta^{ \pm 1}$, and, after a global conjugation by a power of $\alpha$, one can also assume that the last letter of $T$ is not $\alpha^{ \pm 1}$. Then, after expressing $\alpha^{\prime}$ and $\beta^{\prime}$ in terms of $\alpha$ and $\beta$, any reduced word in $\left\{\left(\alpha^{\prime}\right)^{ \pm 1},\left(\beta^{\prime}\right)^{ \pm 1}\right\}$ results in a reduced word: no cancellation occurs. Conversely, there is a word that is equal to $\beta$. Hence, one must have $T=\mathrm{id}$ and $\beta^{\prime}=\beta$.

Proof of Theorem 1.4. Let $g \in \Gamma$ be an element together with a 2-factorization $\overline{\mathfrak{m}}=\left(\mathfrak{m}_{1}, \mathfrak{m}_{2}\right)$. Denote by $G$ the monodromy group of $\overline{\mathfrak{m}}$.

If $G$ is $\Gamma$, then by Lemma 3.1 the pair $\left(\mathfrak{m}_{1}, \mathfrak{m}_{2}\right)$ is conjugate to $\left(R, L^{-1}\right)$, and thus $g$ is conjugate to $\mathbb{X}=R \cdot L^{-1}$, which is an elliptic element.

If $\mathfrak{m}_{1}=\mathfrak{m}_{2}$, then $G$ is a cyclic subgroup of $\Gamma$; hence, $g$ is conjugate to $R^{2}=R \cdot R$, which is a parabolic element.

Otherwise, by Proposition 2.8, $G$ is a proper subgroup such that $G \backslash \Gamma$ is a regular pseudotree $\mathfrak{S}$ with two simple loops. On $\mathfrak{S}$, choose a base point $e$ next to one of the monogons, and fix generators $\alpha, \beta$ of $G=\pi_{1}(\mathfrak{S}, e)$ as shown in Figure 5. By Lemma 3.2, the pair $(\alpha, \beta)$ is weakly Hurwitz equivalent to $\left(\mathfrak{m}_{1}, \mathfrak{m}_{2}\right)$. Therefore, we get $g \sim \mathfrak{m}_{\infty} \sim L^{2} A L^{2} A^{\mathrm{t}}($ see $(2.10))$. If $A=\varnothing$, we get a parabolic element $g \sim L^{4}$; all other elements obtained in this way are hyperbolic.

To finish the proof, note that the three cases mentioned above give the complete list of subgroups generated by two Dehn twists, and the conditions listed in the statement are necessary. For the sufficiency, observe that a factorization $g \sim L^{2} A L^{2} A^{\mathrm{t}}$ is not only a necessary condition but also a description of a particular 2-factorization, with the two Dehn twists as follows:

$$
\begin{aligned}
L^{2} A L^{2} A^{\mathrm{t}} & =\left(\mathbb{X} L^{-1} \mathbb{X}^{-1} \mathbb{Y}\right)(A)\left(\mathbb{X} L^{-1} \mathbb{X}^{-1} \mathbb{Y}\right)\left(\mathbb{Y} A^{-1} \mathbb{Y}\right) \\
& =\mathbb{X} L^{-1} \mathbb{X}^{-1} \cdot(\mathbb{Y} A \mathbb{X}) L^{-1}(\mathbb{Y} A \mathbb{X})^{-1}
\end{aligned}
$$

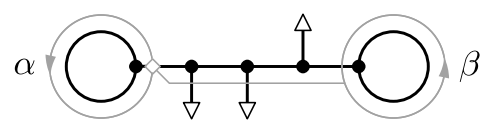

Figure 5: Generators of $G$ with respect to the base point. 


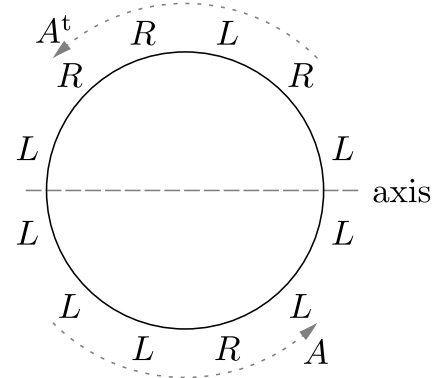

Figure 6: Cyclic diagram associated to $L^{2} A L^{2} A^{\mathrm{t}}$ and its parasymmetry.

Although the converse statements are contained in the above discussions, let us underline the relation between the type of an element and the monodromy group of its 2-factorization.

Corollary 3.4 (of the proof). The monodromy group $G$ of any 2-factorization of an element $g \in \Gamma$ is as follows:

- $g \sim \mathbb{X}$ (elliptic) if and only if $G=\Gamma$;

- $g \sim R^{2}$ (parabolic) if and only if $G \subset \Gamma$ is a cyclic subgroup generated by a single Dehn twist;

- $g \sim L^{4}$ (parabolic) or $g$ is hyperbolic if and only if $G \subset \Gamma$ is a subgroup as in Proposition 2.8.

REMARK 3.5. Geometrically, a representation of an element $g$ in the form (2.10) and the factorization (3.3) can be described in terms of a parasymmetry on the cyclic diagram of $g$. Let us call the four special copies of $L$ in the word $L^{2} A L^{2} A^{\mathrm{t}}$ anchors. On the cyclic diagram, trace an axis passing between the two anchors constituting each of the two pairs $L^{2}$ (see Figure 6). The reflection with respect to this axis preserves the four anchors, while reversing the types of all other letters. A reflection with this property is called a parasymmetry. We underline that the anchors are always of type $L$.

COROLlary 3.6 (of the proof and Remark 2.9). The 2-factorizations (3.3) resulting from two representations $L^{2} A_{1} L^{2} A_{1}^{\mathrm{t}} \sim L^{2} A_{2} L^{2} A_{2}^{\mathrm{t}}$ of the same conjugacy class are weakly equivalent if and only if $A_{1}=A_{2}$ or $A_{1}=A_{2}^{\mathrm{t}}$. $\triangleleft$

\subsection{Proof of Theorem $\mathbf{1 . 5}$}

If $g$ is an elliptic element, we can assume that $g=\mathbb{X}=R \cdot L^{-1}$. Given another 2 -factorization $\mathbb{X}=t_{u} \cdot t_{v}$, the two Dehn twists must generate $\Gamma$ 

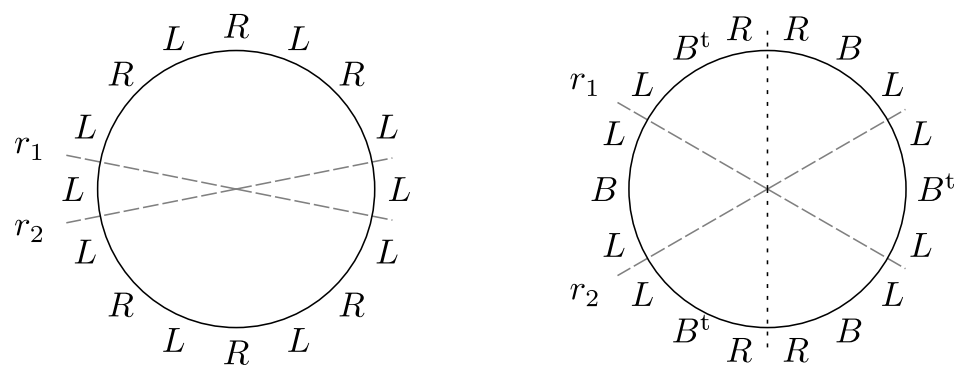

Figure 7: Diagrams with two parasymmetries.

(see Corollary 3.4). Then, due to Lemma 3.1, we have $t_{u}=h^{-1} R h$ and $t_{v}=h^{-1} L^{-1} h$ for some $h \in \Gamma$. It follows that $h$ centralizes $\mathbb{X}$ and hence that $h$ is a power of $\mathbb{X}$ (see Lemma 2.1); thus, the second 2-factorization is strongly equivalent to the first one (as the conjugation by the monodromy at infinity is the Hurwitz move $\sigma_{1}^{-2}$ ).

The only 2-factorization of the parabolic element $g=R^{2}$ is $R^{2}$ itself, as two distinct Dehn twists would produce $\mathbb{X}, L^{4}$, or a hyperbolic element (see Corollary 3.4). Finally, the parabolic element $g \sim L^{4}$ can be regarded as $V_{0}$ (see $(3.7)$ ), and this case is considered below. The two orthogonal parasymmetries of the cyclic diagram of $g$ result in two conjugate (by $L$ ) 2 -factorizations, which are not strongly equivalent, as the corresponding marked skeletons (cf. Figure 8 below) are not isomorphic (see Section 2.5).

Now, assume that $g$ is a hyperbolic element, and consider its cyclic diagram $\mathcal{D}:=\mathcal{D}_{g}$. By assumption, it has two parasymmetries $r_{1}, r_{2}$ (see Remark 3.5); these symmetries generate a certain finite dihedral group $\mathbb{D}_{2 n}$. Let $c:=r_{1} r_{2}$ be the generator of the cyclic subgroup $\mathbb{Z}_{n} \subset \mathbb{D}_{2 n}$; it is the rotation through $2 \alpha$, where $\alpha$ is the angle between the two axes.

3.2.1. The two parasymmetries have a common anchor (see Figure 7, left). In this case, the $\mathbb{D}_{2 n}$-action on $\mathcal{D}$ is obviously transitive, and, starting from an appropriate anchor, we arrive at $g \sim V_{m}$, where $n=2 m+1$ and

$$
V_{m}:=L^{2}(L R)^{m} L^{2}(L R)^{m} \sim L^{2}(R L)^{m} L^{2}(R L)^{m}, \quad m \geq 0 .
$$

In particular, $n$ is odd. It is immediate that $\mathcal{D}$ has no other parasymmetries, as it has only four pairs of consecutive occurrences of $L$, which could serve as anchors. 
3.2.2. The two parasymmetries have no common anchors (see Figure 7, right). Consider the orbits of the $\mathbb{Z}_{n}$-action on $\mathcal{D}$. Call an orbit special or ordinary if it does or does not, respectively, contain an anchor. Each ordinary orbit is "constant," that is, is either $L^{n}$ or $R^{n}$. To analyze a special orbit, start with an anchor $a$ of $r_{1}$ and observe that $c$ preserves the letter $a \uparrow c^{i}$ unless $i=0 \bmod n$ (in this case, $r_{1}$ preserves $a$ and $r_{2}$ reverses $a \uparrow r_{1}$, so that $a \uparrow c$ is an $R$ ) or $i=k:=[n / 2] \bmod n$. In the latter case, if $n=2 k$ is even, then $a \uparrow c^{k}$ is an anchor for $r_{1}$; otherwise, if $n=2 k+1$ is odd, then $a \uparrow c^{k} r_{1}$ is an anchor for $r_{2}$.

Thus, we conclude that $n=2 k+1$ must be odd, as otherwise $a \uparrow c^{k}$, which is an $R$, would be an anchor for $r_{1}$. Furthermore, there are four special orbits of $\mathbb{Z}_{n}$, each one being of the form $L R^{k} L^{k}$ (in the orbit cyclic order, which may differ from the cyclic order restricted from $\mathcal{D}$ ), where the first and the $(k+1)$ st letters are anchors for $r_{1}$ and $r_{2}$, respectively.

Assume that there is a third parasymmetry $r$. Together with $r_{1}$ and $r_{2}$, it generates a dihedral group $\mathbb{D}_{2 m} \supset \mathbb{D}_{2 n}$, and, since $\mathbb{Z}_{n} \subset \mathbb{D}_{2 m}$ is a normal subgroup, $r$ takes $c$-orbits to $c$-orbits, reversing their orbit order. Unless $n=3$, a special orbit is taken to a special one, with one of the two anchors contained in the orbit preserved and the other elements reversed. If $n=3$, a special orbit $L R L$ can be taken to $L^{3}$, with the two copies of $L$ preserved. In both cases, $r$ shares an anchor with $r_{1}$ or $r_{2}$ and $g \sim V_{m}$ for some $m$, which is a contradiction.

Corollary 3.8 (of the proof). In the case of Section 3.2.2, the union of all special orbits is symmetric with respect to the two reflections $s_{1}, s_{2}$ whose axes bisect the angles between $r_{1}$ and $r_{2}$.

Proof. Indeed, since $s_{1} c s_{1}=c^{\prime}:=r_{2} r_{1}$, the orbit $\left\{a_{1} \uparrow c^{i}, i \in \mathbb{Z}\right\}$ starting from an anchor $a_{1}$ of $r_{1}$ is taken (with the letters preserved) to the orbit $\left\{a_{2} \uparrow\left(r_{2} r_{1}\right)^{i}, i \in \mathbb{Z}\right\}$ starting from the anchor $a_{2}:=a_{1} \uparrow s$ of $r_{2}$, and the latter orbit is also special.

The next corollary refines the statement of Theorem 1.5.

Corollary 3.9 (of the proof). The 2-factorizations corresponding to two distinct parasymmetries of the cyclic diagram of a hyperbolic element $g \in \Gamma$ are not weakly equivalent.

Proof. According to Corollary 3.6, the 2-factorizations are weakly equivalent if and only if the two parasymmetries are isomorphic, that is, related by a rotation symmetry of the cyclic diagram. Since the axes cannot be 
orthogonal (see Section 3.2.2), this rotation would give rise to more axes, which would contradict Theorem 1.5.

COROLlARY 3.10. If a hyperbolic 2-factorizable element $g$ is a power $h^{n}$ for some $h \in \Gamma$, then $n=1$ or 2 , and in the latter case, one has $g \sim\left(L^{2} A\right)^{2}$ for a word $A$ in $\{L, R\}$ such that $A^{\mathrm{t}}=A$.

Proof. Under the assumptions, in addition to a parasymmetry $r$, the diagram $\mathcal{D}_{g}$ has a rotation symmetry $c$ of order $n>1$ and hence also parasymmetries $c^{-i} r c^{i}, i=0, \ldots, n-1$. In view of Section 3.2.2, it follows that $n \leq 2$, as otherwise $\mathcal{D}_{g}$ would have two parasymmetries with orthogonal axes (if $n=4$ ) or more than two parasymmetries (if $n=3$ or $n \geq 5$ ).

REMARK 3.11. From Corollary 3.10, it follows immediately that for such $g$, the cutting period cycle is either the minimal period or, at worst, twice the minimal period of the continued fraction expansion.

\subsection{Proof of Theorem $\mathbf{1 . 6}$}

If $g \sim \mathbb{X}$ or $g \sim R^{2}$, the 2-factorization of $g$ is unique up to strong equivalence (see the beginning of Section 3.2).

Let $g$ be a hyperbolic element, and assume that $g=f^{-1} g f$ for some $f \in \Gamma$ that is not a power of $g$. Then both $f$ and $g$ are powers of a hyperbolic element $h \in \Gamma$ (see Lemma 2.1), and, due to Corollary 3.10, we have $g=h^{2} \sim$ $\left(L^{2} A\right)^{2}$ and $A^{\mathrm{t}}=A$ (hence, $\left.\mathbb{Y} A \mathbb{Y}=A^{-1}\right)$. Modulo $g$, we can assume that $f=\left(L^{2} A\right)^{-1}$; then the 2-factorization (3.3) and its conjugate by $f$ differ by one Hurwitz move. (Geometrically, one can argue that the skeleton $\mathfrak{S}$ of the monodromy group has a central symmetry and that the two 2-factorizations are obtained from two symmetric markings of $\mathfrak{S}$.)

Finally, if $g \sim L^{4}$, the corresponding skeleton $\mathfrak{S}$ is as shown in Figure 8. It has four markings with respect to which the monodromy at infinity is $L^{4}$ (see Figure 8), and the corresponding marked skeletons split into two pairs of isomorphic ones, resulting in two strong equivalence classes of 2-factorizations:

$$
L^{4}=R \cdot\left(R^{-1} L^{2}\right) R\left(R^{-1} L^{2}\right)^{-1}=L R L^{-1} \cdot\left(L R^{-1} L^{2}\right) R\left(L R^{-1} L^{2}\right)^{-1} .
$$

Note that the two classes are conjugate by $L$.

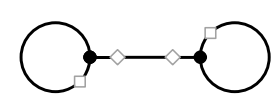

Figure 8: The skeleton corresponding to $g=L^{4}$. 


\subsection{Elements admitting two 2-factorizations}

Let $n=2 k+1$, and consider the word $w_{1 / n}:=l(l r)^{k} l(l r)^{k}$ in the alphabet $\{l, r\}$. Denote by $w[i], i \geq 0$, the $i$ th letter of a word $w$, the indexing starting from 0 . Pick an odd integer $1 \leq m<n$ prime to $n$, and let $w_{q}, q:=m / n$, be the word in $\{l, r\}$ of length $2 n$ defined by

$$
w_{q}[i]=w_{1 / n}[\operatorname{mi} \bmod 2 n], \quad i=0, \ldots, 2 n-1 .
$$

Given a word $B$ in $\{L, R\}$, let $w_{q}\{B\}$ be the word obtained from $w_{q}$ by inserting a copy of $B$ between $w_{q}[2 i]$ and $w_{q}[2 i+1]$ and a copy of $B^{\mathrm{t}}$ between $w_{q}[2 i+1]$ and $w_{q}[2 i+2], i=0, \ldots, n-1$. Finally, let $W_{q}(B)$ be the word obtained from $w_{q}\{B\}$ by the substitution $l \mapsto L^{2}, r \mapsto R^{2}$.

THEOREM 3.12. An element $g \in \Gamma$ admits two distinct strong equivalence classes of 2-factorizations if and only if either $g \sim V_{m}, m \geq 0$ (see (3.7)) or $g \sim W_{q}(B)$, where $0<q<1$ is a rational number with odd numerator and denominator and where $B$ is any word in $\{L, R\}$, possibly empty.

Proof. It has been explained in Section 3.2 that each element $g \sim \mathbb{X}$ or $g \sim R^{2}$ admits a unique 2-factorization, whereas an element $g \sim V_{m}, m \geq 0$, admits two 2-factorizations (which are weakly equivalent if $g \sim L^{4}=V_{0}$; see Section 3.3 for more details on this case). Thus, it remains to consider a hyperbolic element $g$ that is not conjugate to any $V_{m}, m \geq 0$.

Consider the cyclic diagram $\mathcal{D}=\mathcal{D}_{g}$. According to Section 3.2.2, the two parasymmetries $r_{1}$ and $r_{2}$ of $\mathcal{D}$ have no common anchors, and their axes are at an angle $\alpha$ of the form $\pi m / n$, where $n=2 k+1 \geq 3$ is odd and $m$ is prime to $n$. Choosing for $\alpha$ the minimal positive angle and replacing it, if necessary, with $\pi-\alpha$, we can assume that $m$ is also odd and that $0<m<n$, so that $q:=m / n$ is as in the statement. Consider the orbits of the rotation $c:=r_{1} r_{2}$. The union of the special orbits (see Section 3.2.2) is uniquely determined by the angle $\alpha$ : if $m=1$, then $g \sim W_{1 / n}(\varnothing)$ (with the ordinary orbits disregarded; cf. Figure 7, right); otherwise, each orbit is "stretched" $m$ times and "wrapped" back around the circle, so that $g \sim$ $W_{q}(\varnothing)$. In the union of the special orbits, adjacent to each semiaxis of each symmetry contained in $\mathbb{D}_{2 n}$ is a pair of equal letters, either both $L$ or both $R$; these pairs are encoded by, respectively, $l$ and $r$ in the word $w_{1 / n}$ used in the definition of $W_{q}$. These pairs divide the circle into $2 n$ arcs, which are occupied by the ordinary orbits, and, taking into account the full $\mathbb{D}_{2 n}$-action, one can see that the union of all ordinary orbits has the form 
$B, B^{\mathrm{t}}, \ldots, B, B^{\mathrm{t}}$, where $B$ is the portion of this union in one of the arcs (see Figure 7, right); it can be any word in $\{L, R\}$.

\subsection{Relation to real structures}

Here, we discuss the elements of $\Gamma$ that admit both a 2 -factorization and a real structure.

Clearly, an elliptic element $g \sim \mathbb{X}$ and a parabolic element $g \sim R^{2}$ have this property. In both cases, the only 2 -factorization is real. Furthermore, in both cases we have both types of real structures (or real Lefschetz fibrations; see Remark 2.5): for $\mathbb{X}=R \cdot L^{-1}$, the action of $\hat{\tau}_{1}$ preserves the 2 -factorization, whereas that of $\hat{\tau}$ with $\tau=\tau_{2} R^{-1}$ changes it by the Hurwitz move $\sigma_{1}$; for $R^{2}=R \cdot R$, the action of $\hat{\tau}_{2}$ can be regarded as either preserving the 2-factorization or changing it by $\sigma_{1}$.

A parabolic element $g \sim L^{4}$ has two strong equivalence classes of 2-factorizations and four real structures, as can be easily seen from its cyclic diagram. Both 2-factorizations are real with respect to two of the real structures and are interchanged by the two others.

THEOREM 3.13. Assume that a hyperbolic element $g \in \Gamma$ is real and admits a 2-factorization $\overline{\mathfrak{m}}$. If $\overline{\mathfrak{m}}$ is real, then it is unique, and $g$ has a unique real structure. Otherwise, g has two 2-factorizations, both nonreal, which are interchanged by the real structure.

Proof. Under the assumptions, the cyclic diagram $\mathcal{D}:=\mathcal{D}_{g}$ has a parasymmetry (the 2-factorization) $r$ and a symmetry (the real structure) $s$. Then $r^{\prime}:=s r s$ is also a parasymmetry, and unless $r^{\prime}=r$, the two 2-factorizations corresponding to $r$ and $r^{\prime}$ are interchanged by the real structure.

If $r^{\prime}=r$, that is, if $r$ is real, the axes of $r$ and $s$ are orthogonal. (Since $g \nsim L^{4}$, the two axes cannot coincide.) If there were another parasymmetry $r_{1} \neq r$, then $r, r_{1}$, and $r_{1}^{\prime}:=s r_{1} s$ would define three distinct 2-factorizations, which would contradict Theorem 1.5. Similarly, another symmetry $s_{1} \neq s$ would generate, together with $s$, a dihedral group $\mathbb{D}_{2 n}, n \geq 3$, giving rise to $n$ distinct parasymmetries.

REMARK 3.14. The proof of Theorem 3.13 gives us a complete characterization of real hyperbolic elements $g$ admitting a 2-factorization $\overline{\mathfrak{m}}$.

The 2-factorization $\overline{\mathfrak{m}}$ of $g$ is real if and only if $g \sim L^{2} A L^{2} A^{\mathrm{t}}$ for a palindromic word $A$ in $\{L, R\}$.

Otherwise, there are two 2-factorizations, and we have either $g \sim V_{m}$, $m \geq 1$, or $g \sim W_{q}(B)$ (see Theorem 3.12). In the former case, $g$ has two 
real structures, the corresponding symmetries of the cyclic diagram having orthogonal axes. In the latter case, due to Corollary 3.8, the union of the special orbits, that is, the part $W_{q}(\varnothing)$, is symmetric with respect to two reflections $s_{1}, s_{2}$ whose axes are distinguished as those bisecting the "odd" and "even" angles between the axes of the parasymmetries (the horizontal and vertical axes, respectively, in Figure 7). The symmetry $s_{1}$ is a real structure on $W_{q}(B)$ if and only if $B$ is palindromic, whereas $s_{2}$ is a real structure if and only if $B=\varnothing$. (It is worth mentioning that the two nonequivalent factorizations of $V_{n}$ or $W_{q}(B)$ with $B$ palindromic differ by a global conjugation in the group $\operatorname{PGL}(2, \mathbb{Z})$.)

REMARK 3.15. If $\hat{\tau}(\overline{\mathfrak{m}})$ is strongly equivalent to $\overline{\mathfrak{m}}$, then $\hat{\tau}$ preserves the monodromy group $G$ of $\overline{\mathfrak{m}}$ and, hence, induces an orientation reversing symmetry of the skeleton $G \backslash \Gamma$. Clearly, any such symmetry of a skeleton $\mathfrak{S}$ as in Proposition 2.8 with at least one Farey branch must interchange the two monogons of $\mathfrak{S}$. Hence, any real 2 -factorization $\overline{\mathfrak{m}}$ of a hyperbolic element of $\Gamma$ represents a real Lefschetz fibration with a pair of complex conjugate singular fibers (see Remark 2.5); in other words, it is real in the sense that $\hat{\tau}(\overline{\mathfrak{m}})=\overline{\mathfrak{m}}$ for some real structure $\tau$.

\subsection{Further observations}

For practical purposes, the following observation is useful, as it eliminates most matrices as not admitting a 2-factorization.

Proposition 3.16. If an element $g \in \tilde{\Gamma}$ factors into a product of two Dehn twists, then $(2-\operatorname{trace} g)$ is a perfect square.

REMARK 3.17. This is definitely not a sufficient condition; for a counterexample, one can take the element $R^{3} L R^{2}=\left(L^{2} R L^{3}\right)^{\mathrm{t}}$ of trace 7 .

Proof. Up to conjugation, we can assume that the two Dehn twists constituting the product are $R=t_{\mathbf{a}}$ and $A:=t_{[p, q]}$ for some $[p, q] \in \mathcal{H}, \operatorname{gcd}(p, q)=1$. Since

$$
A=\left[\begin{array}{cc}
1-p q & -q^{2} \\
p^{2} & 1+p q
\end{array}\right],
$$

one has trace $R A=2-q^{2}$; on the other hand, trace is a class function.

As a consequence of the proof, we conclude that, for each integer $q$, there does exist an element $g \in \tilde{\Gamma}$ of trace $2-q^{2}$ which is a product of two Dehn twists in $\tilde{\Gamma}$. For an element $g \in \Gamma$, one should check whether $2 \pm \operatorname{trace} g$ is a perfect square. Proposition 3.16 has a geometric meaning: the number 
2 - trace $g$ is the square of the symplectic product of the eigenvectors of the two Dehn twists.

For another necessary condition, consider a finite group $G$, and fix an ordered sequence of conjugacy classes represented by elements $g_{1}, \ldots, g_{r} \in G$. Then the number $N\left(g_{1}, \ldots, g_{r}\right)$ of solutions to the equation $x_{1} \cdot \ldots \cdot x_{r}=\mathrm{id}$, $x_{i} \sim g_{i}, i=1, \ldots, r$, is given by the following Frobenius-type formula (see $[1])$ :

$$
N\left(g_{1}, \ldots, g_{r}\right)=\frac{\left|g_{1}\right| \cdots\left|g_{r}\right|}{|G|} \sum \frac{\chi\left(g_{1}\right) \cdots \chi\left(g_{r}\right)}{\chi(\mathrm{id})^{r-2}},
$$

where $|\cdot|$ stands for the size of the conjugacy class, and the summation runs over all irreducible characters of $G$. Applying this formula to the images of $g_{1}=g_{2}=R, g_{3}=g^{-1}$ in a finite quotient of $\tilde{\Gamma}$, we have the following statement.

Proposition 3.18. If an element $g \in \tilde{\Gamma}$ factors into a product of two Dehn twists, then, for each positive integer $n$, one has

$$
\sum \chi(R)^{2} \chi\left(g^{-1}\right) \chi(\mathrm{id})^{-1} \neq 0
$$

the summation running over all irreducible characters of the group $\operatorname{SL}\left(2, \mathbb{Z}_{n}\right)$.

Note that all irreducible characters of the groups $\operatorname{SL}\left(2, \mathbb{Z}_{p}\right)$ for $p$ prime are known (see, e.g., [13]), and, for each prime $p$, the condition in Proposition 3.18 can be checked effectively in terms of certain Gauss sums. At present, we do not know whether an analogue of the Hasse principle holds for the 2-factorization problem, that is, whether Propositions 3.16 and 3.18 together constitute a sufficient condition for the existence of a 2-factorization.

\section{§4. Real elliptic Lefschetz fibrations}

\subsection{Lefschetz fibrations}

Let $X$ be a compact connected oriented smooth 4-manifold, and let $B$ be a compact connected smooth oriented surface. A Lefschetz fibration is a surjective smooth map $p: X \rightarrow B$ with the following properties:

- $p(\partial X)=\partial B$, and the restriction $p: \partial X \rightarrow \partial B$ is a submersion;

- $p$ has finitely many critical points, which are all in the interior of $X$, and all critical values are pairwise distinct; and

- about each critical point $x$ of $p$, there are local charts $(U, x) \cong\left(\mathbb{C}^{2}, 0\right)$ and $(V, b) \cong\left(\mathbb{C}^{1}, 0\right), b=p(x)$, in which $p$ is given by $\left(z_{1}, z_{2}\right) \mapsto z_{1}^{2}+z_{2}^{2}$. 
The restriction of a Lefschetz fibration to the set $B^{\sharp}$ of regular values of $p$ is a locally trivial fibration with all fibers closed connected oriented surfaces; the genus of $p$ is the genus of a generic fiber. Lefschetz fibrations of genus one are called elliptic.

An isomorphism between Lefschetz fibrations is a pair of orientationpreserving diffeomorphisms of the total spaces and the bases commuting with the projections. The monodromy of a Lefschetz fibration is the monodromy of its restriction to $B^{\sharp}$. As it follows from the local normal form in the definition, the local monodromy (in the positive direction) about a singular fiber is the positive Dehn twist about a certain simple closed curve, well defined up to isotopy; this curve is called the vanishing cycle. The singular fiber itself is obtained from a close nonsingular one by contracting the vanishing cycle to a point to form a single node. A singular fiber is irreducible (remains connected after resolving the node) if and only if its vanishing cycle is not null-homologous. If the vanishing cycle bounds a disk, the singular fiber contains a sphere, which necessarily has self-intersection $(-1)$, that is, is a topological analogue of a $(-1)$-curve. As in the analytic case, such a sphere can be blown down. The fibration is called relatively minimal if its singular fibers do not contain $(-1)$-spheres - that is, none of the vanishing cycles is null-homotopic.

From now on, we consider only relatively minimal elliptic Lefschetz fibrations over the sphere $B=S^{2}$. After choosing a base point $b \in B^{\sharp}$ and fixing an isomorphism $H_{1}\left(p^{-1}(b)\right)=\mathcal{H}$, the monodromy of such a fibration becomes a homomorphism $\pi_{1}\left(B^{\sharp}, b\right) \rightarrow \tilde{\Gamma}$, and it is more or less clear (see [11] for a complete proof) that, up to isomorphism, the fibration is determined by its monodromy. By the Riemann-Hurwitz formula, $\chi(X)=r$, where $r$ is the number of singular fibers.

Theorem 4.1 (Moishezon and Livné (see [11])). Up to isomorphism, a relatively minimal elliptic Lefschetz fibration $X \rightarrow S^{2}$ is determined by the Euler characteristic $\chi(X)$, which is subject to the restrictions $\chi(X) \geq 0$ and $\chi(X)=0 \bmod 12$.

Since for any $k \geq 0$ there exists an elliptic surface $E(k)$ with $\chi(E(k))=$ $12 k$, it follows that any elliptic Lefschetz fibration $p: X \rightarrow S^{2}$ is algebraic (i.e., $X$ and $S^{2}$ admit analytic structures with respect to which $p$ is a regular map).

DEFINITION 4.2. A Jacobian Lefschetz fibration is a relatively minimal elliptic Lefschetz fibration $p: X \rightarrow B \cong S^{2}$ equipped with a distinguished 
section $s: B \rightarrow X$ of $p$. Isomorphisms of such fibrations are required to commute with the sections.

According to Theorem 4.1, any elliptic Lefschetz fibration over $S^{2}$ admits a section, which is unique up to automorphism.

\subsection{Real Lefschetz fibrations}

Mimicking algebraic geometry (cf. Section 5.2 below), define a real structure on a smooth oriented $2 d$-manifold $X$ as an involutive autodiffeomorphism $c_{X}: X \rightarrow X$ with the following properties:

- $c_{X}$ is orientation-preserving (resp., -reversing) if $d$ is even (resp., odd); and

- the real part $X_{\mathbb{R}}:=$ Fix $c_{X}$ is either empty or of pure dimension $d$.

A real Lefschetz fibration is a Lefschetz fibration $p: X \rightarrow B$ equipped with a pair of real structures $c_{X}: X \rightarrow X$ and $c_{B}: B \rightarrow B$ commuting with $p$. Such a fibration is totally real if all its singular fibers are real. (Auto)Homeomorphisms of real Lefschetz fibrations are supposed to commute with the real structures. A Jacobian Lefschetz fibration is real if the distinguished section is real, that is, commutes with the real structures.

Recall that for any real structure $c$ on $X$ one has the Thom-Smith inequality

$$
\beta_{*}\left(X_{\mathbb{R}}\right) \leq \beta_{*}(X),
$$

where $\beta_{*}$ stands for the total Betti number with $\mathbb{Z}_{2}$-coefficients. If (4.3) turns into an equality, the real structure (or the real manifold $X$ ) is called maximal. If $X$ is a closed surface of genus $g$, we have $\beta_{0}\left(X_{\mathbb{R}}\right) \leq g+1$.

From now on, we assume that the base $B$ is the sphere $S^{2}$ and that the real part $B_{\mathbb{R}}$ is a circle $S^{1}$; that is, $c_{B}$ is maximal $\left(B_{\mathbb{R}}\right.$ is sometimes referred to as the equator). A real Lefschetz fibration equipped with a distinguished orientation of $B_{\mathbb{R}}$ is said to be directed; a directed (auto)homeomorphism of such fibrations is an (auto)homeomorphism preserving the distinguished orientations. The fibers over $B_{\mathbb{R}}$ inherit real structures from $c_{X}$; they are called real fibers.

A large supply of real Jacobian Lefschetz fibrations is provided by real Jacobian elliptic surfaces (see Section 5.3). Such fibrations are called algebraic; formally, a real (Jacobian) Lefschetz fibration $p: X \rightarrow B$ is algebraic if $X$ and $B$ admit analytic structures with respect to which $p$ (and $s$ ) are holomorphic and $c_{X}, c_{B}$ are antiholomorphic. It turns out that some (in a sense, most) Lefschetz fibrations are not algebraic; the realizability of a given 
Table 1: Necklace stones.

\begin{tabular}{|c|c|c|c|c|}
\hline Segment & Stone & Dual & Inverse & Monodromy \\
\hline$œ 0$ & $\bigcirc$ & $\square$ & $\bigcirc$ & $\mathbb{Y}^{2} \mathbb{Y}^{2} \mathbb{Y}$ \\
\hline$\Longleftrightarrow \times$ & $\square$ & $\bigcirc$ & $\square$ & $\mathbb{X}^{2} \mathbb{Y} \mathbb{X}^{2}$ \\
\hline$\Longleftrightarrow 0$ & $>$ & $<$ & $<$ & $\mathbb{X} \mathbb{Y}$ \\
\hline$\Leftrightarrow \times$ & $<$ & $>$ & $>$ & $\mathbb{Y} \mathbb{X}$ \\
\hline
\end{tabular}

fibration by an elliptic surface is one of the principal questions addressed in this paper (see Section 6.2).

\subsection{Necklace diagrams}

Define a broken necklace diagram as a nonempty word in the stone alphabet $\{\bigcirc, \square,>,<\}$. Associate to each stone its dual and inverse stones and its monodromy (an element of $\Gamma$ ) as shown in Table 1. Then, given a broken necklace diagram $\mathcal{N}$, we can define its

- monodromy $\mathfrak{m}(\mathcal{N}) \in \Gamma$, which is obtained by replacing each stone with its monodromy and evaluating the resulting word in $\Gamma$;

- dual diagram $\mathcal{N}^{*}$, obtained by replacing each stone with its dual; and

- inverse diagram $\mathcal{N}^{-1}$, obtained by replacing each stone with its inverse and reversing the order of the stones.

Note that the operations of dual and inverse commute with each other and that for any diagram $\mathcal{N}$ one has $\mathfrak{m}\left(\mathcal{N}^{*}\right)=\mathbb{Y} \cdot \mathfrak{m}(\mathcal{N}) \cdot \mathbb{Y}$ and $\mathfrak{m}\left(\mathcal{N}^{-1}\right)=$ $\hat{\tau}_{1}(\mathfrak{m}(\mathcal{N}))$. Furthermore, the symmetric group $\mathbb{S}_{n}$ acts on the set $\operatorname{BND}(n)$ of broken necklace diagrams of length $n$. For any cyclic permutation $\sigma \in \mathbb{S}_{n}$, one has $(\mathcal{N} \uparrow \sigma)^{*}=\mathcal{N}^{*} \uparrow \sigma$ and $(\mathcal{N} \uparrow \sigma)^{-1}=\mathcal{N}^{-1} \uparrow \sigma^{-1}$; thus, on $\operatorname{BND}(n)$ there is a well-defined action of the group $\mathbb{Z}_{2} \times \mathbb{D}_{2 n}$ generated by the dual, inverse, and cyclic permutations.

Definition 4.4. An oriented necklace diagram $\mathcal{N}$ is an element of the quotient set $\operatorname{BND}(n) / \mathbb{Z}_{n}$ by the subgroup $\mathbb{Z}_{n}$ of cyclic permutations or, equivalently, a cyclic word in the stone alphabet. A (nonoriented) necklace diagram is an element of the quotient $\operatorname{BND}(n) / \mathbb{D}_{2 n}$ by the subgroup generated by the cyclic permutations and the inverse.

With real trigonal curves in mind, define also oriented flat and twisted necklace diagrams as elements of the quotients $\operatorname{BND}(n) / \mathbb{Z}_{2} \times \mathbb{Z}_{n}$ and $\operatorname{BND}(n) / \mathbb{Z}_{2} \times \tilde{\mathbb{Z}}_{2 n}$, respectively. Here, $\mathbb{Z}_{2}$ acts via $\mathcal{N} \mapsto \mathcal{N}^{*}, \mathbb{Z}_{n}$ is the subgroup of cyclic permutations, and $\tilde{\mathbb{Z}}_{2 n}$ acts via the twisted shifts $S_{1} S_{2} \cdots S_{n} \mapsto$ 
$S_{2} \cdots S_{n} S_{1}^{*}$. In both cases, the nonoriented versions are defined by further identifying the orbits of $\mathcal{N}$ and $\mathcal{N}^{-1}$.

Consider a directed Jacobian Lefschetz fibration $p: X \rightarrow B$, and assume that it has at least one real singular fiber. The restriction $p_{\mathbb{R}}: X_{\mathbb{R}} \rightarrow B_{\mathbb{R}}$ can be regarded as an $S^{1}$-valued Morse function, and one can assign an index 0,1 , or 2 to each real singular fiber (i.e. the critical point of $p_{\mathbb{R}}$ ). The real part of each real nonsingular fiber is nonempty (as there is a section); hence, it consists of one or two circles (see (4.3)), and the number of circles alternates at each singular fiber. Define the uncoated necklace diagram of $p$ as the following decoration of the oriented circle $B_{\mathbb{R}}$ :

- each singular fiber of index 0 or 2 is marked with $\circ$, and each singular fiber of index 1 is marked with $\times$;

- each segment connecting two consecutive singular fibers over which nonsingular fibers have two real components is doubled.

A typical real part $X_{\mathbb{R}}$ and its uncoated necklace diagram are shown in Figure 9, middle and bottom, respectively.

Definition 4.5. The oriented necklace diagram $\mathcal{N}(p)$ of a directed Jacobian Lefschetz fibration $p: X \rightarrow B$ is the cyclic word in the stone alphabet obtained by replacing each double segment of its uncoated necklace diagram with a single stone, as shown in Table 1 . In the presence of a base point
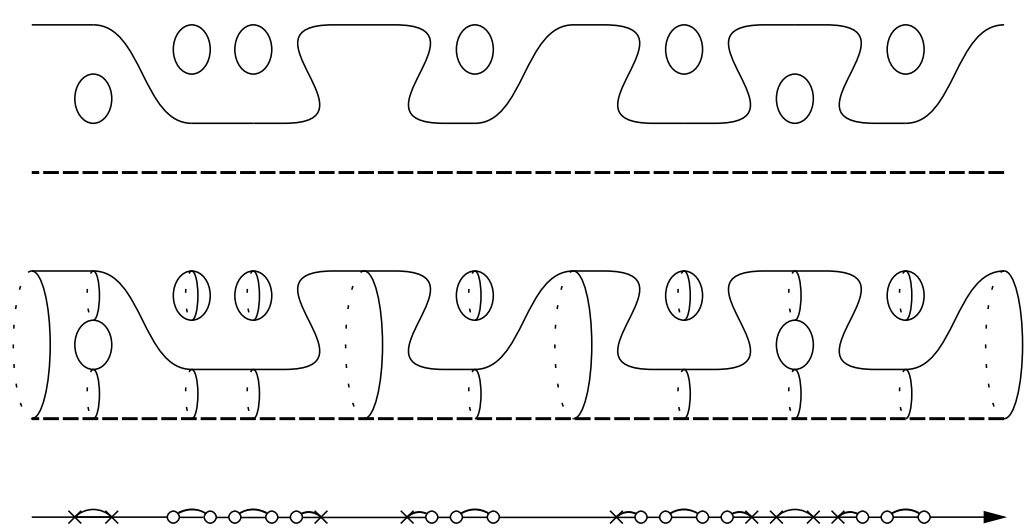

Figure 9: A nonhyperbolic trigonal curve (top), a covering Jacobian surface (middle), and its uncoated necklace diagram (bottom); the horizontal dashed lines represent the distinguished sections. 
$b$ inside one of the simple segments of $B_{\mathbb{R}}$, one can also speak about the broken necklace diagram $\mathcal{N}_{b}(p)$ of $p$, with the convention that the first stone $S_{1}$ is the immediate successor of $b$.

For example, the necklace diagram of the fibration shown in Figure 9 is

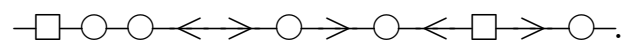

(In [19], necklace diagrams are drawn in the oriented circle $B_{\mathbb{R}}$, and we respect this convention by drawing a "broken" necklace. For long diagrams we will also use the obvious multiplicative notation for associative words.) According to the following theorem, a totally real fibration is uniquely recovered from its necklace diagram.

THEOREM 4.6 (see [20], [19]). Given $k>0$, the map $p \mapsto \mathcal{N}(p)$ establishes a bijection between the set of isomorphism classes of (directed) totally real Jacobian Lefschetz fibrations with $12 k$ singular fibers and the set of (oriented) necklace diagrams of length $6 k$ and monodromy id $\in \Gamma$.

The classification of totally real Lefschetz fibrations for the small values of $k$ is also found in [20] and [19]. For $k=1$, there are 25 undirected isomorphism classes, among which four are maximal. For $k=2$, the number of classes is 8421 .

\subsection{Generalizations}

Let $\mathcal{N}$ be a broken necklace diagram. A w-pendant on $\mathcal{N}$ is a strong Hurwitz equivalence class of $w$-factorizations $\overline{\mathfrak{m}}$ of $\mathfrak{m}(\mathcal{N})$. The $\left(\mathbb{Z}_{2} \times \mathbb{D}_{2 n}\right)$ action on the set $\operatorname{BND}(n)$ is extended to pairs $(\mathcal{N}, \overline{\mathfrak{m}})$ as follows:

- the inverse $(\mathcal{N}, \overline{\mathfrak{m}})^{-1}$ is $\left(\mathcal{N}^{-1}, \hat{\tau}_{1}(\overline{\mathfrak{m}})\right)$;

- the dual $(\mathcal{N}, \overline{\mathfrak{m}})^{*}$ is $\left(\mathcal{N}^{*}, \mathbb{Y} \overline{\mathfrak{m}} \mathbb{Y}\right)$;

- the cyclic permutation $1 \mapsto 2 \mapsto \cdots$ acts via $\mathcal{N}=S_{1} \cdots S_{n} \mapsto S_{2} \cdots S_{n} S_{1}$ and $\overline{\mathfrak{m}} \mapsto P_{1}^{-1} \overline{\mathfrak{m}} P_{1}$, where $P_{1}$ is the monodromy of $S_{1}$.

An oriented $w$-pendant necklace diagram is an orbit of the cyclic permutation action on the set of pairs $(\mathcal{N}, \overline{\mathfrak{m}})$ as above; a (nonoriented) $w$-pendant necklace diagram is obtained by the further identification of the orbits of $(\mathcal{N}, \overline{\mathfrak{m}})$ and $(\mathcal{N}, \overline{\mathfrak{m}})^{-1}$. The length of a $w$-pendant necklace diagram represented by $(\mathcal{N}, \overline{\mathfrak{m}})$ is the length $|\mathcal{N}|$, the number of stones on $\mathcal{N}$.

REMARK 4.7. An oriented flat $w$-pendant necklace diagram is defined as an orbit of the further action $(\mathcal{N}, \overline{\mathfrak{m}}) \mapsto(\mathcal{N}, \overline{\mathfrak{m}})^{*}$. In the case of twisted necklace diagrams, both the monodromy and the notion of $w$-pendant should be 
defined slightly differently. Namely, given a broken necklace diagram $\mathcal{N}$, let $\tilde{\mathfrak{m}}(\mathcal{N}):=\mathfrak{m}(\mathcal{N}) \mathbb{Y}$. The twisted shift by the cyclic permutation $\sigma: 1 \mapsto 2 \mapsto \cdots$ acts via $\tilde{\mathfrak{m}}(\mathcal{N} \uparrow \sigma)=P_{1}^{-1} \tilde{\mathfrak{m}}(\mathcal{N}) P_{1}$, and we can define a twisted $w$-pendant as a strong equivalence class of $w$-factorizations $\overline{\mathfrak{m}}$ of $\tilde{\mathfrak{m}}(\mathcal{N})$. The twisted action of $\mathbb{Z}_{2} \times \tilde{\mathbb{Z}}_{2 n}$ extends to pairs $(\mathcal{N}, \overline{\mathfrak{m}})$ in the same way as above, and an oriented twisted w-pendant necklace diagram is defined as an orbit set of this action. The nonoriented analogues are defined as above, by the further identification of the orbits of $(\mathcal{N}, \overline{\mathfrak{m}})$ and $(\mathcal{N}, \overline{\mathfrak{m}})^{-1}$.

Let $p: X \rightarrow B$ be a directed Jacobian Lefschetz fibration with $r>0$ real and $w \geq 0$ pairs of complex conjugate singular fibers. Denote by $B_{+} \subset B$ the closed hemisphere inducing the chosen orientation of the equator $B_{\mathbb{R}}$. Decorate $B_{\mathbb{R}}$ as explained in Section 4.3, and remove from $B_{+}$the union of some disjoint regular neighborhoods of the stones (i.e., double segments); denote the resulting closed disk by $\Omega$, and let $\Omega^{\sharp}=\Omega \cap B^{\sharp}$. Choose a base point $b \in \Omega \cap B_{\mathbb{R}}$, and pick a geometric basis $\left\{\delta_{1}, \ldots, \delta_{w}\right\}$ for the group $\pi_{1}\left(\Omega^{\sharp}, b\right)$ (see Figure 10, where black dots denote nonreal singular fibers).

The real structure $c:=\left.c_{X}\right|_{F_{b}}$ in the real fiber $F_{b}$ over $b$ is conjugate to $\tau_{1}$; it gives rise to a distinguished pair of opposite bases $\pm(\mathbf{a}, \mathbf{b})$ in the homology $H_{1}\left(F_{b}\right)$, which are defined by the condition that $\mathbf{a} \pm \mathbf{b}$ should be a $( \pm 1)$-eigenvector of $c_{*}$. Thus, there is a canonical, up to sign, identification $H_{1}\left(F_{b}\right)=\mathcal{H}$, and the monodromies $\mathfrak{m}\left(\delta_{i}\right)$ project to well-defined elements $\mathfrak{m}_{i} \in \Gamma, i=1, \ldots, w$. Let $\overline{\mathfrak{m}}_{b}(p)=\left(\mathfrak{m}_{1}, \ldots, \mathfrak{m}_{w}\right)$.

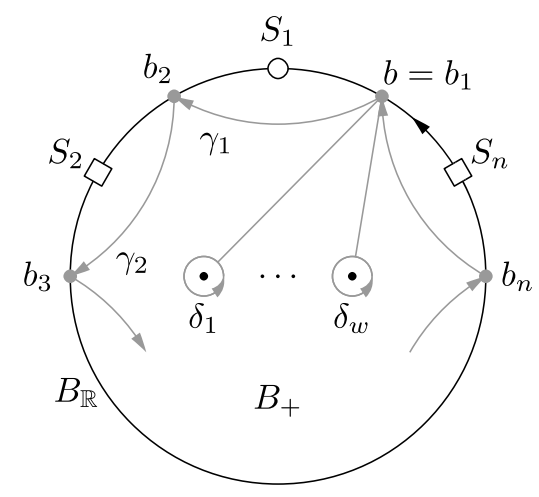

Figure 10: The monodromy of a real Lefschetz fibration. 
LEMMA 4.8. The strong equivalence class of the $w$-factorization $\overline{\mathfrak{m}}_{b}(p)$ is indeed a $w$-pendant on the broken necklace diagram $\mathcal{N}_{b}(p)$. A change of the base point $b$ used in the definition results in a cyclic permutation action on the pair $\left(\mathcal{N}_{b}, \overline{\mathfrak{m}}_{b}\right)$.

Proof. According to [20], the monodromy $P_{i}$ of a stone $S_{i}$ is the $\Gamma$-valued monodromy along a path $\gamma_{i}$ connecting two points $b_{i}$ and $b_{i+1}$, right before and right after $S_{i}$, and circumventing $S_{i}$ in the clockwise direction (see Figure 10). (To obtain a well-defined element of $\Gamma$, in the fibers over both points one should use the canonical bases described above.) Hence, the first statement of the lemma follows from the obvious relation $\gamma_{1} \cdot \ldots \cdot \gamma_{n} \sim[\partial \Omega]$. For the second statement, it suffices to notice that, changing the base point from $b=b_{1}$ to $b_{2}$, one can take for a new geometric basis for $\pi_{1}\left(\Omega^{\sharp}, b_{2}\right)$ the set $\left\{\gamma_{1}^{-1} \delta_{i} \gamma_{1}\right\}, i=1, \ldots, w$.

Theorem 4.9. The map sending $p: X \rightarrow B$ to the class of the pair $\left(\mathcal{N}_{b}(p), \overline{\mathfrak{m}}_{b}(p)\right)$ establishes a bijection between the set of isomorphism classes of (directed) Jacobian Lefschetz fibrations with $2 n>0$ real and $w$ pairs of complex conjugate singular fibers and the set of (oriented) w-pendant necklace diagrams of length $n$.

Proof. Due to Lemma 4.8, the map in question is well defined, and to complete the proof it suffices to show that a Lefschetz fibration can be recovered from a pair $(\mathcal{N}, \overline{\mathfrak{m}})$ uniquely up to isomorphism. The necklace diagram $\mathcal{N}$ gives rise to a unique, up to isomorphism, totally real directed Jacobian Lefschetz fibration over an equivariant regular neighborhood $U$ of the equator $B_{\mathbb{R}}$ (see [20] for details; this statement is an essential part of the proof of Theorem 4.6). The complement $B \backslash U$ consists of two connected components $B_{ \pm}^{\circ}$, and $\overline{\mathfrak{m}}$ is a $w$-factorization of the monodromy $\mathfrak{m}\left(\partial B_{+}^{\circ}\right)=$ $\mathfrak{m}(\mathcal{N})$; due to [11], this factorization determines a unique extension of the fibration from $\partial B_{+}^{\circ}$ to $B_{+}^{\circ}$. The extension to the other half $B_{-}^{\circ}$ is defined by symmetry.

REMARK 4.10. It is not easy to decide whether a given necklace diagram $\mathcal{N}$ admits a $w$-pendant. There are simple criteria for $w=0$ (one must have $\mathfrak{m}(\mathcal{N})=\mathrm{id}), w=1(\mathfrak{m}(\mathcal{N})$ must be a Dehn twist), and $w=2$ (the criterion is given by Theorem 1.4). In general, one can lift $\mathfrak{m}(\mathcal{N})$ to a degree $w$ element in the braid group $\mathbb{B}_{3}$ and apply Orevkov's quasi-positivity criterion (see [15]): a $w$-pendant exists if and only if the lift is quasi-positive. A lift of degree $w$ exists (and then is unique) if and only if $\operatorname{deg} \mathfrak{m}(\mathcal{N})=w \bmod 6$, 
where deg : $\Gamma \rightarrow \mathbb{Z}_{6}$ is the abelianization epimorphism, with the convention that $\operatorname{deg} R=1$. Obviously, this condition is necessary for the existence of a $w$-pendant.

REMARK 4.11. If $w=0$ or 1 , a necklace diagram $\mathcal{N}$ obviously admits at most one $w$-pendant. If $w=2$, there are at most two $w$-pendants (see Theorems 1.5 and 3.12). It follows that at most two isomorphism classes of real Jacobian Lefschetz fibrations with two pairs of complex conjugate singular fibers may share the same necklace diagram (equivalently, fibered topology of the real part).

We used Maple to compute the numbers of undirected isomorphism classes of real Jacobian Lefschetz fibrations for some small values of $k$ and $w$ (where $12 k$ is the total number of singular fibers and $w$ is the number of pairs of complex conjugate ones). For $k=1$, the numbers are $25(w=0), 28(w=1)$, and $24(w=2)$; for $k=2$, they are $8421(w=0)$ and $15602(w=1)$. (For $k=w=2$, the computation is too long.) In all examples, a fibration with $w>0$ pairs of conjugate singular fibers can be obtained from one with $(w-1)$ pairs by converting the pair of real fibers constituting an arrowtype stone to a pair of conjugate ones. We do not know how general this phenomenon is.

\subsection{Counts}

We conclude this section with a few simple counts. Let $p: X \rightarrow B$ be a real Jacobian Lefschetz fibration, $\chi(X)=12 k>0$, and let $\mathcal{N}=\mathcal{N}(p)$. We assume that $\mathcal{N} \neq \varnothing$, so that $0<|\mathcal{N}| \leq 6 k$. Denote by $\#_{\diamond}:=\#_{\diamond}(\mathcal{N})$ the number of stones of type $\diamond, \diamond \in\{\bigcirc, \square,>,<\}$. Then one has

$$
\beta_{*}(X)=\chi(X)=12 k
$$

and

$$
\beta_{*}\left(X_{\mathbb{R}}\right)=2\left(\#_{\bigcirc}+\# \square\right)+4, \quad \chi\left(X_{\mathbb{R}}\right)=2\left(\#_{\circ}-\# \square\right)
$$

(see [20], [19], or Figure 9). In particular, $\#_{\bigcirc}+\#_{\square} \leq 6 k-2$ (see (4.3)), and $X$ is maximal if and only if $\#_{\bigcirc}+\# \square=6 k-2$. Alternatively, $X$ is maximal if and only if

$$
\#<+\#>+w=2
$$

where $w \leq 2$ is the number of pairs of complex conjugate singular fibers. 


\section{$\S 5$. Real trigonal curves}

\subsection{Trigonal curves}

A Hirzebruch surface $\Sigma_{d}, d>0$, is a geometrically ruled rational surface with a (unique) exceptional section $E$ of self-intersection $-d$. We denote the ruling by $p: \Sigma_{d} \rightarrow B \cong \mathbb{P}^{1}$; its fibers are called the fibers of $\Sigma_{d}$. A (proper) trigonal curve is a reduced curve $C \subset \Sigma_{d}$ disjoint from the exceptional section and intersecting each fiber at three points (counted with multiplicities); in other words, the restriction $p: C \rightarrow B$ is a map of degree 3. A singular fiber of a trigonal curve is a fiber of $\Sigma_{d}$ intersecting the curve geometrically at fewer than three points; equivalently, singular are the fibers over the critical values of the restriction $\left.p\right|_{C}$. In this paper, we consider almost generic trigonal curves only; that is, we assume that all critical points of $\left.p\right|_{C}$ are simple. Such a curve is nonsingular and irreducible; hence, it has genus $g(C)=3 d-2$ (the adjunction formula), and the number of singular fibers is $6 d$ (the Riemann-Hurwitz formula).

Given a point $b \in B$, let $F_{b}$ be the fiber $p^{-1}(b)$, and let $F_{b}^{\circ}$ be the affine fiber $F_{b} \backslash E$. It is an affine complex line. Hence, in the presence of a trigonal curve, one can speak about the zero section $Z$ sending each point $b \in B$ to the barycenter of the three points $C \cap F_{b}^{\circ}$ and about the fiberwise convex hull conv $C \subset \Sigma_{d} \backslash E$.

\subsection{Real trigonal curves}

Recall that a real structure on an algebraic (analytic) variety $X$ is an antiholomorphic involution $c_{X}: X \rightarrow X$. A pair $\left(X, c_{X}\right)$ is called a real algebraic variety; usually, the real structure $c$ is understood, and we speak about a real algebraic variety $X$. Given a real structure, the fixed point set $X_{\mathbb{R}}:=$ Fix $c_{X}$ is called the real part of $X$. A maximal real algebraic variety (see (4.3)) is usually called an $M$-variety.

Up to isomorphism, a Hirzebruch surface $\Sigma_{d}$ admits a unique real structure $c_{d}$ with nonempty real part $\left(\Sigma_{d}\right)_{\mathbb{R}}$, the latter being a torus or a Klein bottle for $d$ even or odd, respectively. This real structure $c_{d}$ descends to a certain real structure $c_{B}$ on the base $B$, so that the ruling $p$ is real. In what follows, when speaking about a real Hirzebruch surface, we assume such a pair $\left(c_{d}, c_{B}\right)$ fixed. Any real automorphism $\varphi: \Sigma_{d} \rightarrow \Sigma_{d}$ induces a $c_{B}$-equivariant autohomeomorphism (in fact, a real automorphism) $\varphi_{B}: B \rightarrow B$. Such an autohomeomorphism $\varphi_{B}$ (and the original automorphism $\varphi$ ) is said to be 
directed if it preserves the orientation of $B_{\mathbb{R}}$. More generally, a directed autohomeomorphism of a real Hirzebruch surface $p: \Sigma_{d} \rightarrow B$ is an orientationpreserving $c_{d}$-equivariant fiberwise autohomeomorphism whose descent to $B$ preserves the orientations of $B$ and $B_{\mathbb{R}}$ (cf. Section 4.2).

A trigonal curve $C \subset \Sigma_{d}$ is real if it is $c_{d}$-invariant; then, the restriction of $c_{d}$ to $C$ is a real structure on $C$. In affine coordinates, such a curve is given by a polynomial with real coefficients. By a deformation of real trigonal curves, we mean an equivariant deformation (a path in the space of real polynomials) in the class of almost generic curves. Two curves are said to be (directedly) deformation equivalent if they differ by a deformation and/or a (directed) real automorphism of $\Sigma_{d}$.

Fix a real trigonal curve $C \subset \Sigma_{d}$, and consider the restriction $p_{\mathbb{R}}: C_{\mathbb{R}} \rightarrow$ $B_{\mathbb{R}}$. If each fiber of $p_{\mathbb{R}}$ consists of three points, the curve $C$ is called hyperbolic. In this case, the real part $C_{\mathbb{R}}$ consists of three (if $d$ is even) or two (if $d$ is odd) components; in the former case, each component is mapped onto $B_{\mathbb{R}}$ homeomorphically, and in the latter case, one "central" component is mapped homeomorphically and the other is a double covering. It can be shown that all hyperbolic curves (in a given surface) are deformation equivalent (see, e.g., [7]). If $C$ is not hyperbolic, its real part looks like that shown in Figure 9, top. More precisely, $C_{\mathbb{R}}$ has one long component that is mapped onto $B_{\mathbb{R}}$ and, possibly, a number of ovals, necessarily unnested. The long component may contain some zigzags (the Z-shaped fragments in Figure 9). For a formal definition, consider a maximal, with respect to inclusion, segment $I \subset B_{\mathbb{R}}$ with the property that each point $b \in I$ has at least two pullbacks under $p_{\mathbb{R}}$. If the pullback $p_{\mathbb{R}}^{-1}(I)$ is disconnected, one of its components is an oval; otherwise, the pullback is called a zigzag. With a certain abuse of the language, the projections of the ovals and zigzags to $B_{\mathbb{R}}$ (i.e., maximal segments $I \subset B_{\mathbb{R}}$ as above) are also referred to as ovals and zigzags, respectively (cf. Section 5.5 below).

\subsection{The covering elliptic surface}

Let $C \subset \Sigma_{d}$ be a real trigonal curve. If $d$ is even, the double covering $X \rightarrow \Sigma_{d}$ ramified at $C+E$ is a Jacobian elliptic surface ( $E$ being the section), and the real structure $c_{d}$ lifts to two opposite real structures $c_{ \pm}: X \rightarrow X$ that differ by the deck translation of the covering. Disregarding the analytic structure, one can consider the corresponding real varieties $X_{ \pm}$ as real Jacobian Lefschetz fibrations. 
The necklace diagrams $\mathcal{N}\left(X_{ \pm}\right)$are dual to each other, and each of them determines the embedded topology of the real part $C_{\mathbb{R}} \subset\left(\Sigma_{d}\right)_{\mathbb{R}}$. Hence, the latter can be encoded by the pair $\mathcal{N}\left(X_{ \pm}\right)$, that is, by a flat necklace diagram.

If $d$ is odd, a covering elliptic surface exists only over the complement of the fiber $F_{b}$ over a point $b \in B$. In this case, choosing for $b$ a generic real point with one preimage under $p_{\mathbb{R}}$ and analyzing the dependence of $X_{\mathbb{R}}$ on $b$, one can see that the real part $C_{\mathbb{R}} \subset\left(\Sigma_{d}\right)_{\mathbb{R}}$ is encoded by a twisted necklace diagram.

In both cases, the ovals of $C_{\mathbb{R}}$ correspond to the $\square$ - and $\bigcirc$-type stones, whereas the zigzags correspond to the arrow-type stones (see Figure 9).

\subsection{Dessins}

In appropriate (real) affine coordinates $(x, y)$, a (real) trigonal curve $C \subset$ $\Sigma_{d}$ can be given by its Weierstraß equation

$$
y^{3}+g_{2}(x) y+g_{3}(x)=0
$$

where $g_{2}$ and $g_{3}$ are some (real) polynomials in $x$ of degree at most $2 d$ and $3 d$, respectively, and the discriminant $\Delta(x):=-4 g_{2}^{3}-17 g_{3}^{2}$ is not identically zero. The $j$-invariant of $C$ is defined as the meromorphic function

$$
j_{C}: B \rightarrow \mathbb{P}^{1}=C \cup\{\infty\}, \quad j_{C}:=-\frac{4 g_{2}^{3}}{\Delta} .
$$

If $C$ is real, so is $j_{C}$.

If $C$ is almost generic, by a small equisingular deformation it can be made generic, that is, such that the $j$-invariant $j_{C}$ has generic branching behavior in the sense of [11], which means that all zeros of $j_{C}$ are triple, all zeros of $\left(j_{C}-1\right)$ are double, all poles of $j_{C}$ are simple, and all critical values of $j_{C}$ other than 0 or 1 are also simple. (We emphasize that these properties are highly nongeneric for a map $B \rightarrow \mathbb{P}^{1}$, but they do correspond to truly generic trigonal curves.) For generic real trigonal curves, we define (directed) strict deformation equivalence as the equivalence relation generated by the (directed) real automorphisms of $\Sigma_{d}$ and equivariant equisingular deformations in the class of generic curves.

Fix a generic trigonal curve $C$, and define its dessin $\mathfrak{D}:=\operatorname{Dssn} C$ as the embedded graph $j_{C}^{-1}\left(\mathbb{P}_{\mathbb{R}}^{1}\right) \subset B$ decorated as follows:

- the pullbacks of 0,1 , and $\infty$ are $\bullet$-, o-, and $\times$-vertices, respectively;

- an edge is colored solid, bold, or dotted if its image belongs to $(-\infty, 0)$, $(0,1)$, or $(1, \infty)$, respectively, and is directed according to the canonical orientation of $\mathbb{P}_{\mathbb{R}}^{1}$, that is, the standard linear order on $\mathbb{R}$; 
- the critical points of $j_{C}$ with real critical values distinct from 0,1 , or $\infty$ are considered monochrome vertices, respectively, solid, bold, or dotted. If $C$ is real, its dessin $\mathfrak{D}$ is invariant with respect to the real structure $c$ on $B$, and the real part $B_{\mathbb{R}}$ is a union of edges and vertices of $\mathfrak{D}$. The properties of the graph thus obtained are summarized in the following definition.

Definition 5.3. Let $B$ be the sphere $S^{2}$, and let $c: B \rightarrow B$ be the reflection against the equator. A (real) dessin is a $c$-invariant embedded directed graph $\mathfrak{D} \subset B$ decorated with the following additional structures (referred to as the colorings of the edges and vertices of $\mathfrak{D}$, respectively)

- each edge of $\mathfrak{D}$ is of one of the three kinds: solid, bold, or dotted; and

- each vertex of $\mathfrak{D}$ is of one of the four kinds: $\bullet, \circ, \times$, or monochrome (the vertices of the first three kinds being called essential);

and satisfying the following conditions:

(1) the equator of $B$ is a union of edges and vertices of $\mathfrak{D}$, and each monochrome vertex is at the equator;

(2) each •-, o-, ×-, or monochrome vertex has valency $6,4,2$, or 4 , respectively;

(3) the orientations of the edges of $\mathfrak{D}$ induce an orientation of the boundary of the complement $B \backslash \mathfrak{D}$;

(4) all edges incident to a monochrome vertex are of the same kind;

(5) the $\times$-vertices are incident to incoming dotted edges and outgoing solid edges;

(6) the •-vertices are incident to incoming solid edges and outgoing bold edges;

(7) the o-vertices are incident to incoming bold edges and outgoing dotted edges;

(8) $\mathfrak{D}$ has no directed monochrome cycles, that is, directed cycles with all edges of the same kind and all vertices monochrome.

In items (5)-(7), the lists are complete, that is, vertices cannot be incident to edges of other kinds or with a different orientation.

The equator Fix $c$ is called the real part of the dessin; the edges and vertices of $\mathfrak{D}$ that are in the equator are called real, whereas the other vertices are called inner.

Two dessins are said to be (directedly) homeomorphic if they are related by a (directed) orientation-preserving $c$-equivariant autohomeomorphism of $B$. Note that directedly homeomorphic dessins are, in fact, equivariantly isotopic. 


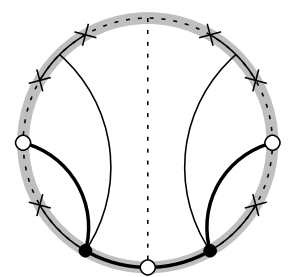

(a) $I_{2}$

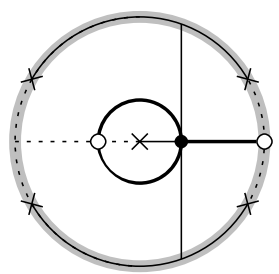

(b) $\mathrm{I}_{1}$

Figure 11: Dessins of $M$-cubics.

If $C$ is a generic curve in $\Sigma_{d}$, then $\operatorname{deg} j_{C}=6 d$, and hence, the numbers of •-, ०-, and $\times$-vertices of the dessin $\operatorname{Dssn} C$ are $2 d, 3 d$, and $6 d$, respectively. The number $3 d$ of o-vertices is called the degree of the curve $C$ and dessin $\operatorname{Dssn} C$.

Convention. In view of the symmetry, in the figures we draw only the portion of a dessin contained in one of the two hemispheres, which is represented by a disk. The real part of the dessin (the boundary of the disk) is shown by a thick gray line (cf., e.g., Figure 11). When speaking about directed dessins, we choose the closed hemisphere $B_{+}$whose orientation induces the fixed orientation of the equator $B_{\mathbb{R}}$.

Theorem 5.4 (see [7], [14]). The map $C \mapsto D \operatorname{Dsn} C$ establishes a bijection between the set of (directed) strict deformation equivalence classes of generic real trigonal curves and that of (directed) homeomorphism classes of dessins.

REMARK 5.5. The notion of strict deformation equivalence is not very meaningful from the topological point of view, as some codimension 1 degenerations of the $j$-invariant do not affect the topology of the curve. It is shown in [7] that deformation equivalence classes of almost generic curves are in a one-to-one correspondence with certain equivalence classes of dessins, where two dessins are considered equivalent if they are related by a sequence of homeomorphisms and certain elementary moves. We omit the description of these moves as they are not essential in the case of $M$-curves, which are the only ones considered in this paper.

Definition 5.6. The union of the bold edges and •- and o-vertices of the dessin $\operatorname{Dssn} C$ is called the skeleton of $C$ and is denoted by $\operatorname{Sk} C$. If $\operatorname{Dssn} C$ has no bold monochrome vertices, $\mathrm{Sk} C$ is a regular skeleton in the sense of the definition given in Section 2.5. 


\subsection{Topology in terms of dessins}

The topology of the real part $C_{\mathbb{R}} \subset\left(\Sigma_{d}\right)_{\mathbb{R}}$ is easily recovered from the $\operatorname{dessin} \mathfrak{D}:=\operatorname{Dssn} C$. It is immediate from (5.1), (5.2), and the definition of $\mathfrak{D}$ that

(1) the singular fibers of $C$ are those over the $\times$-vertices of $\mathfrak{D}$;

(2) the points of intersection $C \cap Z$ are over the o-vertices of $\mathfrak{D}$; and

(3) a point $b$ inside a real edge $e$ of $\mathfrak{D}$ has three preimages under $p_{\mathbb{R}}$ if and only if $e$ is dotted.

It follows that the ovals and zigzags of $C_{\mathbb{R}}$, regarded as subsets of $B_{\mathbb{R}}$, are the maximal dotted segments in $B_{\mathbb{R}}$; any such segment is bounded by two $\times$-vertices and is allowed to contain a number of o- or monochrome vertices inside. In view of item (2) above, a maximal dotted segment in $B_{\mathbb{R}}$ is an oval (resp., zigzag) if and only if it contains an even (resp., odd) number of o-vertices.

The uncoated necklace diagram $N$ of the covering elliptic surface $X$ of $C$ is also recovered from $\mathfrak{D}$. (If the degree of $C$ is odd, we should fix a base point $b$ inside a solid or bold real edge of $\mathfrak{D}$ and speak about the uncoated diagram broken at $b$.) The $\times$ - and o-points of $N$ are the $\times$-vertices of $\mathfrak{D}$, and the double segments of $N$ are the maximal dotted segments of $\mathfrak{D}$. (At this point, we need to apologize for the notation clash. Unfortunately, both notation sets are quite well established, and it seems unwise to change them.) Since $X$ has two opposite real structures, we cannot distinguish between the $\times$ - and o-type critical points, but we can compare pairs of points: two critical points are of the same type (both $\times$ or both $\circ$ ) if and only if they are separated by an even number of o-vertices of $\mathfrak{D}$. All assertions are simple consequences of elementary Morse theory; they are obvious from Figure 9.

Given a necklace diagram $\mathcal{N}$, denote by $\#_{\text {ess }}$ the number of simple segments of the corresponding uncoated diagram connecting pairs of critical points of opposite types, that is, those of the form $\circ \rightarrow x$ or $x \rightarrow 0$. (Such segments are called essential.) Since any real Jacobian elliptic surface $X$ is the double covering of the Hirzebruch surface $\Sigma_{d}=X / \pm$ id ramified at $E$ and a certain real trigonal curve $C$, we have the following necessary condition for a necklace diagram $\mathcal{N}$ to be algebraic.

Proposition 5.7 (see [20], [19]). Let $X \rightarrow B$ be a real Jacobian elliptic surface with $\chi(X)=12 k>0$. Then its necklace diagram $\mathcal{N}$ is subject to the inequalities

$$
\#_{\mathrm{ess}} \leq 2 k, \quad \#_{\mathrm{ess}}+\#_{<}+\#_{>} \leq 6 k
$$


Proof. The second statement follows from the fact that each zigzag (an arrow-type stone) and each essential segment contains an odd number, hence at least one, of $\circ$-vertices of the dessin, and $\#_{\circ}=6 k$. For the first one, observe that a o-vertex inside an essential segment is separated from each of the two $\times$-vertices bounding the segment by at least one •-vertex (as the type of the edges must change from bold to solid; cf. Figure 11), and $\# \bullet=4 k$.

\subsection{The monodromy}

Let $\Omega \subset B$ be a closed disk. A continuous section $s: \Omega \rightarrow \Sigma_{d}$ of $p$ is called proper (with respect to a fixed trigonal curve $C$ ) if its image is disjoint from both $E$ and $\operatorname{conv} C$. Since the disk $\Omega$ is contractible and all fibers $F_{b} \backslash(E \cup \operatorname{conv} C)$ are connected, a proper section exists and is unique up to homotopy in the class of proper sections.

Fix a trigonal curve $C$, a disk $\Omega$, and a proper section $s$. Assume that the boundary $\partial \Omega$ contains no singular fibers of $C$, and denote by $\Omega^{\sharp}$ the disk $\Omega$ with all singular fibers removed. The restriction $p: p^{-1}\left(\Omega^{\sharp}\right) \backslash(C \cup E) \rightarrow \Omega^{\sharp}$ is a locally trivial fibration, and one can consider the associated bundle with the discrete fibers Aut $\pi_{1}\left(F_{x} \backslash(C \cup E), s(x)\right), x \in \Omega^{\sharp}$. This bundle is a covering, and, fixing a base point $b \in \Omega^{\sharp}$ and lifting loops starting from the identity over $b$, we obtain a homomorphism $\tilde{\mathfrak{m}}: \pi_{1}\left(\Omega^{\sharp}, b\right) \rightarrow$ Aut $\pi_{b}$, where $\pi_{b}:=\pi_{1}\left(F_{b} \backslash(C \cup E), s(b)\right)$. This homomorphism is called the monodromy; since the section $s$ is proper, it actually takes values in the braid group $\mathbb{B}_{3} \subset$ Aut $\pi_{b}$, where $\pi_{b}$ is identified with the free group $\mathbb{F}_{3}$ by means of a geometric basis.

We will "downgrade" the monodromy to the modular group $\Gamma$ and consider the composition $\mathfrak{m}: \pi_{1}\left(\Omega^{\sharp}, b\right) \rightarrow \mathbb{B}_{3} \rightarrow \Gamma$, called the reduced monodromy. If $d$ is even, $\mathfrak{m}$ coincides with the $\Gamma$-valued reduction of the monodromy (homological invariant) of the covering elliptic surface. The following statement is essentially contained in [5], although the conventions and notation in [5] differ slightly from those used in this paper.

THEOREM 5.8 (see [5]). Consider a connected component $\mathfrak{S}_{0}$ of the intersection $\mathrm{Sk} C \cap \Omega$, and assume that the base point $b$ is in an edge ef $\mathfrak{S}_{0}$. Then, under an appropriate choice of a geometric basis in the reference fiber $F_{b}$, the diagram 


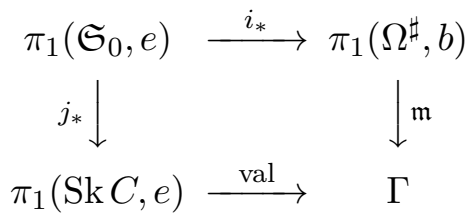

commutes, where $i: \mathfrak{S}_{0} \hookrightarrow \Omega^{\sharp}$ and $j: \mathfrak{S}_{0} \hookrightarrow$ Sk $C$ are the inclusions.

Now, assume that the curve $C$ is real. Orient the real part $B_{\mathbb{R}}$, and consider the positive hemisphere $B_{+}$. Choose some disjoint regular neighborhoods $U_{i} \subset B$ of the singular fibers of $C$, and let $\Omega=B_{+} \backslash \bigcup U_{i}$, the union running over the real singular fibers only (cf. a similar construction in Section 4.4 and Figure 10). Pick a base point $b$ in the boundary $\partial \Omega$, make the other necessary choices, and consider the reduced monodromy $\mathfrak{m}: \pi_{1}\left(\Omega^{\sharp}, b\right) \rightarrow \Gamma$.

Definition 5.9. The image $\operatorname{MG}(C):=\operatorname{Im} \mathfrak{m} \subset \Gamma$ is called the monodromy group of $C$; the element $\mathfrak{m}_{\infty}:=\mathfrak{m}[\partial \Omega] \in \operatorname{MG}(C)$ is called the monodromy at infinity.

The following statement is straightforward.

Proposition 5.10. The pair $\left(\mathrm{MG}, \mathfrak{m}_{\infty}\right), \mathfrak{m}_{\infty} \in \mathrm{MG} \subset \Gamma$, is determined by the curve $C$ and orientation of $B_{\mathbb{R}}$ up to conjugation. The conjugacy class of the pair $\left(\mathrm{MG}, \mathfrak{m}_{\infty}\right)$ is invariant under directed autohomeomorphisms of the pair $\left(\Sigma_{d}, C\right)$; in particular, it is a directed deformation invariant of real trigonal curves.

The conjugacy class of the monodromy at infinity depends on the real part $C_{\mathbb{R}}$ only. Indeed, choose the base point $b$ real and outside the zigzags and ovals, and represent the real part by a broken (at $b$ ) necklace diagram $\mathcal{N}$, flat or twisted (see Section 5.3). Then, up to conjugation, one has $\mathfrak{m}_{\infty}=\mathfrak{m}(\mathcal{N})$ if $d$ is even and $\mathfrak{m}_{\infty}=\tilde{\mathfrak{m}}(\mathcal{N})$ if $d$ is odd. (Note that the conjugacy class of $\tilde{\mathfrak{m}}(\mathcal{N})$ is preserved by the twisted shifts used in the definition of twisted diagrams (see Remark 4.7).)

\subsection{Real trigonal $M$-curves}

In view of (4.3), the real part $C_{\mathbb{R}}$ of an $M$-curve $C \subset \Sigma_{d}$ has $3 d-1$ connected components. Hence, unless $d=1$, such a curve is nonhyperbolic, its real part has $3 d-2$ ovals, and, since each oval and each zigzag consumes two real singular fibers, one has $z+w=2$, where $z$ is the number of zigzags and 
$w$ is the number of pairs of complex conjugate singular fibers; in particular, $z, w \leq 2$. Comparing this with (4.14), we conclude that $C$ is an $M$-curve if and only if any/both covering elliptic surfaces $X_{ \pm}$are $M$-varieties. (This statement trivially holds for hyperbolic curves as well.)

For $d=1$ (essentially, plane cubics), there are four deformation families of $M$-curves: one hyperbolic and three nonhyperbolic, denoted by $\mathrm{I}_{z}, z=0,1,2$ : a curve of type $\mathrm{I}_{z}$ has one oval and $z$ zigzags (see Figure 11 for $z=1$ and 2).

To describe the other $M$-curves, we need the operation of junction. Consider two directed dessins $\mathfrak{D}^{i} \subset B^{i}, i=1,2$. Choose a pair of zigzags $Z^{i}$ of $\mathfrak{D}^{i}$ with a single o-vertex $v^{i}$ in each, and let $I^{i} \subset \partial B_{+}^{i}$ be a segment contained in the interior of $Z^{i}$ and containing $v^{i}$ inside. Pick a homeomorphism $\varphi: I^{1} \rightarrow I^{2}$, and consider the connected boundary sum $B_{+}:=B_{+}^{1} \sqcup_{\varphi} B_{+}^{2}$ and the graph

$$
\mathfrak{D}_{+}:=\left(\mathfrak{D}^{1} \cap B_{+}^{1}\right) \sqcup_{\varphi}\left(\mathfrak{D}^{2} \cap B_{+}^{2}\right) \subset B_{+} .
$$

Finally, double $B_{+}$to form a new sphere $B \cong S^{2}$, and double $\mathfrak{D}_{+}$to form a graph $\mathfrak{D} \subset B$; the two real o-vertices $v^{1}, v^{2}$ are replaced with a pair of complex conjugate o-vertices, and the common endpoints of $I^{1}, I^{2}$ become monochrome vertices of $\mathfrak{D}$. The resulting graph $\mathfrak{D} \subset B$ is a dessin; it is called the junction of $\mathfrak{D}^{1}$ and $\mathfrak{D}^{2}$ along the pair of zigzags $Z^{1}, Z^{2}$. Up to isotopy, the junction depends only on the pair of dessins $\mathfrak{D}^{1}, \mathfrak{D}^{2}$ and the pair of zigzags $Z^{1}, Z^{2}$ and on whether the homeomorphism $\varphi$ is orientationpreserving or -reversing. The zigzags $Z^{1}, Z^{2}$ are "consumed" by the junction, being replaced by a pair of ovals. It follows that an iterated junction of several dessins does not depend on the order of the individual operations: one can start with the disjoint union of all dessins involved and identify all pairs of segments (which are all disjoint) simultaneously.

An example of junction is shown in Figure 12, where only some essential parts of the dessin are drawn.

THEOREM 5.11 (see [7], [14]). Each directed deformation class of real trigonal $M$-curves $C \subset \Sigma_{d}, d \geq 2$, contains a representative whose dessin is

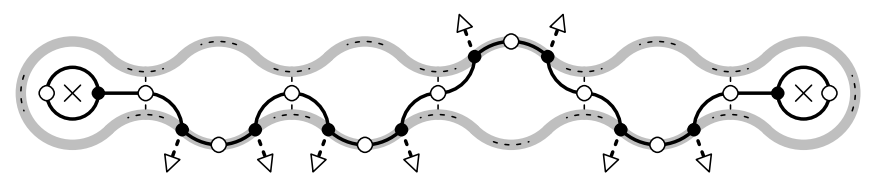

Figure 12: A junction of six $M$-cubics $(* \uparrow \uparrow \downarrow \uparrow *)$. 
an iterated junction of $d$ copies of cubic dessins $\mathrm{I}_{2}, \mathrm{I}_{1}$ shown in Figure 11. Conversely, any such junction is the dessin of an $M$-curve. Two (directed) deformation classes are equal if and only if their corresponding dessins as above are (directedly) homeomorphic.

According to the number of zigzags, a dessin of type $\mathrm{I}_{1}$ participates in exactly one junction (consuming its only zigzag), whereas a dessin of type $\mathrm{I}_{2}$ can participate in one or two junctions. Furthermore, a dessin of type $\mathrm{I}_{1}$ has a "horizontal" axis of symmetry preserving the zigzag (see Figure 11), whereas a dessin of type $\mathrm{I}_{2}$ does not. It follows that, after the junction as in Theorem 5.11, the individual blocks form a linear chain, which can be encoded by a word in the alphabet $\{\uparrow, \downarrow, *\}$, with the convention that $*$ can only appear as the first and/or last letter. Here, * represents a dessin of type $\mathrm{I}_{1}$, and $\uparrow$ and $\downarrow$ represent a dessin of type $\mathrm{I}_{2}$, oriented, respectively, as shown in Figure 11 or upside down (see Figure 12 for an example). On the set $M_{d}$ of such words of length $d$, there is an action of the group $\mathbb{Z}_{2} \times \mathbb{Z}_{2}$ generated by the vertical flip $v$, reversing the order of the letters, and the horizontal flip $h$, interchanging $\uparrow$ and $\downarrow$. Each flip is realized by a homeomorphism of dessins reversing the orientation of the equator. In these terms, one can restate Theorem 5.11 as follows.

Corollary 5.12. The directed (resp., undirected) deformation classes of real trigonal $M$-curves in $\Sigma_{d}$ are in a natural one-to-one correspondence with the orbit set $M_{d} / v h$ (resp., the orbit set $M_{d} /\langle v, h\rangle$ ).

The number of $*$-type letters in the word representing an $M$-curve $C$ equals the number $w \leq 2$ of pairs of complex conjugate singular fibers of $C$.

As explained at the beginning of this section, an $M$-curve $C$ has at most two zigzags. If $C$ has at least one zigzag, the appropriate (flat or twisted) necklace diagram of $C$ determines the representation of the $\operatorname{dessin} \operatorname{Dssn} C$ in the form of iterated junction and, hence, the deformation class of $C$ (see [7] for details). If $C$ has no zigzags, this assertion is no longer true. The shortest example is the pair of degree 12 curves represented by $* \uparrow \downarrow *$ and $* \downarrow \uparrow *$ : they share the same oriented flat necklace diagram $\bigcirc^{5} \square^{5}$ but are not related by a directed deformation equivalence. The two degree 30 curves represented by

$$
* \downarrow \uparrow \downarrow \downarrow \uparrow \uparrow \downarrow \uparrow * \quad \text { and } \quad * \downarrow \uparrow \uparrow \downarrow \uparrow \downarrow \downarrow \uparrow *
$$

share the same oriented diagram $\left(\bigcirc^{5} \square^{5} \bigcirc \square^{3}\right)^{2}$ but are not deformation equivalent, directedly or not. An explanation of this phenomenon is given 
in Remark 6.7 below. We address this (non)uniqueness question in greater detail in the next section (see Corollaries 6.5 and 6.6).

\section{§6. Geometric applications}

\subsection{Real trigonal $M$-curves are quasi-simple}

Recall that a deformation family of complex algebraic varieties is called quasi-simple if, within this family, the equivariant deformation class of a real variety is determined by the topology of its real structure. The first geometric application of our algebraic results is the quasi-simplicity of real trigonal $M$-curves.

Theorem 6.1. Two real trigonal $M$-curves $C_{1}, C_{2} \subset \Sigma_{d}$ are in the same (directed) deformation class if and only if the quadruples $\left(\Sigma_{d}, C_{i}, p, c_{d}\right), i=$ 1,2 , are related by a (directed) homeomorphism.

The case where each curve has at least one zigzag is settled in [7]; in this case, nonequivalent curves differ by their real parts (more precisely, appropriate necklace diagrams, flat or twisted). Thus, we need to consider curves without zigzags only (equivalently, those with two pairs of conjugate singular fibers), and for such curves Theorem 6.1 follows from a much stronger statement, Theorem 6.3 below.

Definition 6.2. A word $w$ in the alphabet $\{L, R\}$ is called even if all letters occur in $w$ in pairs or, equivalently, if $w$ can be represented as a word in $\left\{L^{2}, R^{2}\right\}$. A parabolic or hyperbolic element $g \in \Gamma$ is even if, up to conjugation, $g$ is represented by an even word in $\{L, R\}$. For a hyperbolic element, this condition is equivalent to the requirement that all entries of the cutting period cycle of $g$ should be even. Finally, a regular pseudotree $\mathfrak{S}$ with two loops (cf. Proposition 2.8) is even if its monodromy at infinity is even. Informally, $\mathfrak{S}$ is even if its Farey branches pointing upward/downward appear in pairs (see Remark 2.9).

Theorem 6.3. Two real trigonal $M$-curves $C^{\prime} \subset \Sigma_{d^{\prime}}$ and $C^{\prime \prime} \subset \Sigma_{d^{\prime \prime}}$ without zigzags are directedly deformation equivalent (in particular, $d^{\prime}=d^{\prime \prime}$ ) if and only if the monodromy groups $\mathrm{MG}\left(C^{\prime}\right)$ and $\mathrm{MG}\left(C^{\prime \prime}\right)$ are conjugate in $\Gamma$. Furthermore, a subgroup $G \subset \Gamma$ is the monodromy group of a real trigonal $M$-curve without zigzags if and only if the skeleton $G \backslash \Gamma$ is an even regular pseudotree with two loops.

Proof. Let $C$ be a trigonal curve as in the statement. According to Theorem 5.11, we can assume that the dessin of $C$ is a junction of two cubic 
dessins $I_{1}$ and several cubic dessins $I_{2}$ (see Figure 12). (To simplify the figure, we show only the real part $B_{\mathbb{R}}$, maximal real dotted segments (which are all ovals), junctions, and the portion of the skeleton $\operatorname{Sk} C$ that is in $B_{+}$.) We extend the graph $\mathrm{Sk} C \cap B_{+}$to a regular skeleton $\mathfrak{S}^{\prime}$ by attaching, at each real •-vertex, a Farey branch reaching beyond the boundary to $B_{-}$ (shown as thick dashed lines in Figure 12). Then, the following statements are straightforward.

- The skeleton $\mathfrak{S}^{\prime}$ is an even regular pseudotree with two loops (cf. Proposition 2.8).

- Any even regular pseudotree with two loops can be obtained in this way, starting from a certain junction of $M$-cubics.

- The monodromy group $\mathrm{MG}(C)$ is the stabilizer of $\mathfrak{S}^{\prime}$ (see Theorem 5.8).

- The dessin Dssn $C$ is uniquely recovered from $\mathfrak{S}^{\prime}$.

The first two statements imply the last assertion of the theorem. The fact that a curve is uniquely determined by its monodromy group follows from the last two statements and Theorem 5.11.

In view of our previous results concerning 2-factorizations, we have the following corollaries of Theorem 6.3 .

Corollary 6.4 (cf. Theorem 1.4). A necklace diagram $\mathcal{N}$ (flat or twisted) without arrow-type stones is the diagram of a real trigonal $M$-curve if and only if the monodromy $\mathfrak{m}(\mathcal{N})$ has the form $L^{2} A L^{2} A^{\mathrm{t}}$ for some even word $A$ in $\{L, R\}$.

Corollary 6.5 (cf. Theorem 1.5). A necklace diagram $\mathcal{N}$ as in Corollary 6.4 is the diagram of at most two, up to equivalence, real trigonal $M$-curves. In other words, at most two equivalence classes of curves may have homeomorphic real parts.

Corollary 6.6 (cf. Theorem 1.5). A necklace diagram $\mathcal{N}$ as in Corollary 6.4 gives rise to two equivalence classes of real trigonal $M$-curves if and only if the monodromy $\mathfrak{m}(\mathcal{N})$ has the form $W_{q}(B)$ for some even word $B$ in $\{L, R\}$.

Remark 6.7. The two $M$-curves given by (5.13) which share the same topology of the real part (i.e., flat necklace diagram) correspond to the two nonequivalent 2-factorizations of the element $W_{1 / 3}\left(L^{2} R^{2}\right)$ (see Section 3.4 and Theorem 3.12). 


\subsection{Maximal Lefschetz fibrations are algebraic}

As stated in the introduction, one of the major questions in the theory of real elliptic Lefschetz fibrations is whether a given fibration can be realized by an algebraic one. Unlike the complex case, there do exist nonalgebraic real Lefschetz fibrations. Thus, among the 25 undirected isomorphism classes of totally real fibrations with 12 singular fibers, only 17 are algebraic (see [20], [19]); the eight others are ruled out by Proposition 5.7. Of the 8421 classes of totally real fibrations with 24 singular fibers, at least 4825 classes are nonalgebraic as they violate Proposition 5.7. At present, we do not know if all 3596 remaining classes are algebraic, nor do we know any simple criterion that would establish that a given fibration is algebraic. (In the case of 12 singular fibers, an analytic structure is constructed in [20] and [19] by finding a dessin with the desired flat necklace diagram.)

In this section, we prove Theorem 1.8, closing the question for maximal fibration.

Consider a fibration $p: X \rightarrow B$ as in the statement of Theorem 1.8, and let $\mathcal{N}:=\mathcal{N}(p)$ be its necklace diagram. Let $w$ be the number of pairs of complex conjugate singular fibers of $p$; we have $w \leq 2$ (see (4.14)). The case $w=0$ is covered by Theorem 1.7 , and it remains to consider the cases $w=1,2$.

Proof of Theorem 1.8: The case $w=2$. The diagram $\mathcal{N}$ has no arrowtype stones (see (4.14)); hence, $\mathcal{N}$ is of the form $\square^{i_{1}} \bigcirc^{j_{1}} \cdots \square^{i_{s}} \bigcirc^{j_{s}}$ (assuming that $\mathcal{N}$ has both $\bigcirc$ - and $\square$-type stones and breaking it between a $\bigcirc$ and a $\square$ ). Then, using Table 1, after cancellations we have

$$
\mathfrak{m}(\mathcal{N})=\left(R L^{i_{1}-1} R\right)\left(R L^{j_{1}-1} R\right) \cdots\left(R L^{i_{s}-1} R\right)\left(R L^{j_{s}-1} R\right) .
$$

Thus, in the cyclic diagram $\mathcal{D}$ of $\mathfrak{m}(\mathcal{N})$, the copies of $R$ appear in pairs. On the other hand, since $\mathfrak{m}(\mathcal{N})$ admits a 2 -factorization (see Theorem 4.9), $\mathcal{D}$ has a parasymmetry (see Remark 3.5). It follows that the copies of $L$ also appear in pairs (two pairs of anchors and pairs of $L$ symmetric to those of $R)$; that is, $\mathfrak{m}(\mathcal{N})$ is an even element of $\Gamma$. Hence, the monodromy group of any 2 -factorization of $\mathfrak{m}(\mathcal{N})$ is as in Theorem 5.11, and the corresponding flat pendant necklace diagram is realized by a real trigonal $M$-curve $C$. Due to Theorem 4.9, one of the two opposite real Jacobian elliptic surfaces ramified at $C+E$ is isomorphic to $p$.

If all stones of $\mathcal{N}$ are of the same type, then, since the monodromy of each $\bigcirc$ - or $\square$-type stone is conjugate to $L$ (see Table 1 ), we have $\mathfrak{m}(\mathcal{N}) \sim$ 
$L^{r}$, where $r$ is the length of $\mathcal{N}$. Hence, $r=4$ and $\mathcal{N}=\bigcirc^{4}$ or $\square^{4}$ (see Theorem 1.4), and in view of Theorem 1.6, $\mathcal{N}$ admits two nonequivalent 2-pendants. It is immediate that the four real Lefschetz fibrations obtained in this way are isomorphic to the four rational real elliptic surfaces ramified over the two zigzag-free trigonal $M$-curves of degree 6 , namely, $* \uparrow \uparrow *$ and $* \uparrow \downarrow *$.

To complete the proof, we need a few observations and a lemma.

Consider a real Jacobian elliptic $M$-surface $p: X \rightarrow B$ with two pairs of conjugate singular fibers. In the class of real Jacobian elliptic surfaces, there are exactly four ways to collide a pair of conjugate singular fibers to a single real fiber $F$ of type $\tilde{\mathbf{A}}_{0}^{* *}$ (Kodaira's type II) and perturb $F$ to produce a pair of real singular fibers (see, e.g., [7]; in the realm of real trigonal $M$-curves, this procedure corresponds to replacing one of the $*$-type letters at an end of the word representing the junction of $M$-cubics with $\uparrow$ or $\downarrow$ ). In two cases, the new necklace diagram has an extra stone of type $>$, and in the two other cases, it has an extra stone of type $<$. Cutting the original diagram $\mathcal{N}(p)$ at a base point $b$ right before the new stone, we conclude that the pendant necklace diagram of $p$ has two representatives of the form $\left(\mathcal{N}^{\prime},\left(R, g^{\prime}\right)\right.$ ) (for some $g^{\prime} \in \Gamma$ ) and two representatives of the form $\left(\mathcal{N}^{\prime \prime},\left(L^{-1}, g^{\prime \prime}\right)\right.$ ) (for some $\left.g^{\prime \prime} \in \Gamma\right)$.

Conversely, given a representative of a pendant necklace diagram of the form, for example, $(\mathcal{N},(R, \ldots))$, the corresponding real Lefschetz fibration can be modified, in the topological category, so that a pair of conjugate singular fibers disappears to produce a >-type stone. (Topologically, we merely remove an equivariant disk surrounding the two fibers and replace it with another disk, with two real singular fibers and the same monodromy at the boundary; see the discussion of the two real structures on the 2-factorization $\mathbb{X}=R \cdot L^{-1}$ in Section 3.5.) The following lemma asserts that, in the case of a maximal Lefschetz fibration, any such topological modification is one of the two described above and hence can be realized in the algebraic category.

LEMMA 6.8. The pendant necklace diagram of a real Jacobian elliptic $M$-surface with two pairs of complex conjugate real fibers has exactly two representatives of the form $\left(\mathcal{N}^{\prime},\left(R, g^{\prime}\right)\right.$ ) (for some $g^{\prime} \in \Gamma$ ) and exactly two representatives of the form $\left(\mathcal{N}^{\prime \prime},\left(L^{-1}, g^{\prime \prime}\right)\right.$ ) (for some $\left.g^{\prime \prime} \in \Gamma\right)$.

Proof. We consider the representatives of the form $\left(\mathcal{N}^{\prime},\left(R, g^{\prime}\right)\right)$, which result in the $>$-type stones. According to the discussion above, two such 
representatives do exist; they correspond to the two algebraic modifications of the fibration. Fix one of these representatives. Since the action of $\sigma_{1}^{2}$ is the conjugation by $R g^{\prime}=\mathfrak{m}\left(\mathcal{N}^{\prime}\right)$, the 2-factorization of any other representative in question is either $\left(P^{-1} R P, \ldots\right)$ or $\left(P^{-1} g^{\prime} P, \ldots\right)$, where $P$ is a monodromy (not necessarily the shortest, i.e., possibly multiplied by a power of $\mathfrak{m}\left(\mathcal{N}^{\prime}\right)$ ) from the original base point $b^{\prime}$ to the new base point $b^{\prime \prime}$. We assert that $P^{-1} R P=R$ if and only if $P=\mathrm{id}$. Indeed, consider the part $\operatorname{Sk} C \cap B_{+}$ of the skeleton of the corresponding real trigonal curve and extend it to a pseudotree $\mathfrak{S}$ as explained in the proof of Theorem 6.3 (cf. Figure 12). Assume that the original base point $b^{\prime}$ is contained in the boundary of the leftmost cubic dessin $\mathrm{I}_{1}$ in Figure 12, and assign to $b^{\prime}$ the base point $e^{\prime}:=e \uparrow \mathbb{Y}$ of $\mathfrak{S}$, where $e$ is the edge constituting the leftmost monogonal region. Starting from $e^{\prime}$, "project" any other base point $b^{\prime \prime}$ to $\mathfrak{S}$ by assigning to $b^{\prime \prime}$ the edge $e^{\prime \prime}:=e^{\prime} \uparrow P$. Taking into account Table 1 and using induction, one can easily show that the edges obtained are either as shown in Figure 13 (if the stone $S$ preceding $b^{\prime}$ is of type $\square$ ) or those in the figure shifted by $\mathbb{Y}$ (if $S$ is of type $\bigcirc$ ). Crucial is the fact that all these points are pairwise distinct. On the other hand, $e^{\prime} \uparrow R^{n}=e^{\prime}$ for any $n \in \mathbb{Z}$. Hence, unless $P=\mathrm{id}$, the monodromy $P$ is not a power of $R$, and we have $P^{-1} R P \neq R$.

It follows, in particular, that the $>$-type stone that may appear in the cubic dessin $\mathrm{I}_{1}$ at the other end of the junction results in a 2-factorization of the other form, that is, $\left(P^{-1} g^{\prime} P, \ldots\right)$, and the same argument as above shows that this representative is also unique.

Proof of Theorem 1.8: The case $w=1$. According to (4.14), the necklace diagram $\mathcal{N}:=\mathcal{N}(p)$ has a single arrow-type stone $S$, which we can assume to be of type $>$. (Otherwise, switch to the dual diagram $\mathcal{N}^{*}$.) Replace the two real singular fibers contained in $S$ with a pair of conjugate singular

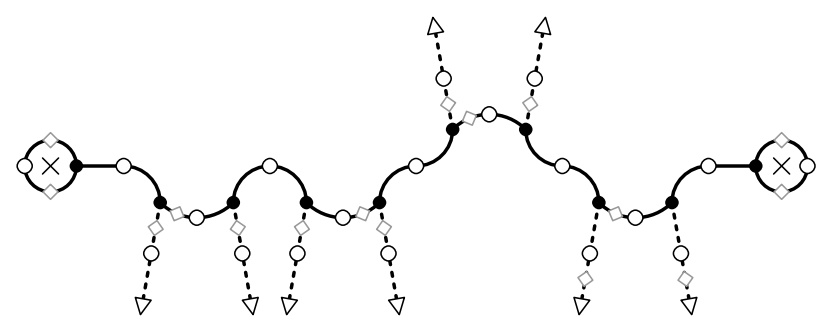

Figure 13: Necklace base points in the skeleton. 
fibers (the inverse of the operation described prior to Lemma 6.8). The new Jacobian Lefschetz fibration is maximal and, according to the first part of the proof, is algebraic. Now, due to Lemma 6.8 , there are only two ways to revert the operation and recreate a >-type stone; both result in algebraic Lefschetz fibrations.

As a consequence of Theorem 1.8, we have a more effective description of the deformation classes of maximal real Jacobian Lefschetz fibrations: the directed (resp., undirected) deformation classes of such fibrations are in a natural one-to-one correspondence with the orbit set $M_{d} / v h$ (resp., the orbit set $\left.M_{d} /\langle v, h\rangle\right)$ (cf. Corollary 5.12).

Acknowledgments. This paper was essentially completed during the second author's stay as a Leibniz fellow and the first author's visit as a Forschungsgast at the Mathematisches Forschungsinstitut Oberwolfach; we are grateful to this institution and its friendly staff for their hospitality and for the excellent working conditions. We would like to thank Viatcheslav Kharlamov for his encouragement and interest in the subject, and Alexander Klyachko, who brought to our attention the Frobenius-type formulas counting solutions to equations in finite groups. We are also grateful to Anton Klyachko and to the anonymous referee of this text, who drew our attention to Bardakov's paper [3] and Kulkarni's paper [9], respectively.

\section{REFERENCES}

[1] Z. Arad, J. Stavi, and M. Herzog, "Powers and products of conjugacy classes in groups" in Products of Conjugacy Classes in Groups, Lecture Notes in Math. 1112, Springer, Berlin, 1985, 6-51. MR 0783068. DOI 10.1007/BFb0072286.

[2] E. Artin, Theory of braids, Ann. of Math. (2) 48 (1947), 101-126. MR 0019087.

[3] V. G. Bardakov, The structure of a group of conjugating automorphisms (in Russian), Algebra Logika 42 (2003), no. 5, 515-541, 636; English translation in Algebra Logic 42 (2003), no. 5, 287-303. MR 2025714. DOI 10.1023/A:1025913505208.

[4] F. Bogomolov and Y. Tschinkel, "Monodromy of elliptic surfaces" in Galois Groups and Fundamental Groups, Math. Sci. Res. Inst. Publ. 41, Cambridge University Press, Cambridge, 2003, 167-181. MR 2012216.

[5] A. Degtyarev, Zariski k-plets via dessins d'enfants, Comment. Math. Helv. 84 (2009), 639-671. MR 2507257. DOI 10.4171/CMH/176.

[6] - Hurwitz equivalence of braid monodromies and extremal elliptic surfaces, Proc. Lond. Math. Soc. (3) 103 (2011), 1083-1120. MR 2861751. DOI 10.1112/ plms/pdr013.

[7] A. Degtyarev, I. Itenberg, and V. Kharlamov, On deformation types of real elliptic surfaces, Amer. J. Math. 130 (2008), 1561-1627. MR 2464028. DOI 10.1353/ajm.0. 0029. 
[8] V. S. Kulikov, D. Oru, and V. Shevchishin, Regular homotopy of Hurwitz curves (in Russian), Izv. Ross. Akad. Nauk Ser. Mat. 68 (2004), no. 3, 91-114; English translation in Izv. Math. 68 (2004), no. 3, 521-542. MR 2069195. DOI 10.1070/ IM2004v068n03ABEH000487.

[9] R. S. Kulkarni, An arithmetic-geometric method in the study of the subgroups of the modular group, Amer. J. Math. 113 (1991), 1053-1133. MR 1137534. DOI 10.2307/ 2374900.

[10] R. Matveyev and K. Rafi, personal communication, 2011.

[11] B. Moishezon, Complex Surfaces and Connected Sums of Complex Projective Planes, with an appendix by R. Livne, Lecture Notes in Math. 603, Springer, Berlin, 1977. MR 0491730.

[12] - "The arithmetic of braids and a statement of Chisini" in Geometric Topology (Haifa, 1992), Contemp. Math. 164, Amer. Math. Soc., Providence, 1994, 151-175. MR 1282761. DOI 10.1090/conm/164/01591.

[13] M. A. Nămark and A. I. Štern, Theory of Group Representations, Grundlehren Math. Wiss. 246, Springer, New York, 1982. MR 0793377. DOI 10.1007/978-1-4613-8142-6.

[14] S. Y. Orevkov, Riemann existence theorem and construction of real algebraic curves, Ann. Fac. Sci. Toulouse Math. (6) 12 (2003), 517-531. MR 2060598.

[15] —, Quasipositivity problem for 3-braids, Turkish J. Math. 28 (2004), 89-93. MR 2056762.

[16] - On braid monodromy monoid, presentation given at Mathematical Sciences Research Institute, April 2004.

[17] F. Öztürk and N. Salepci, Real open books and real contact structures, preprint, arXiv:1202.5928 [math.GT]

[18] N. Salepci, Real elements in the mapping class group of $T^{2}$, Topology Appl. 157 (2010), 2580-2590. MR 2719402. DOI 10.1016/j.topol.2010.06.012.

[19] - Classification of totally real elliptic Lefschetz fibrations via necklace diagrams, J. Knot Theory Ramifications 21 (2012), no. 9. MR 2926572.

[20] — Real Lefschetz fibrations, Ph.D. dissertation, Université Louis Pasteur, Strasbourg, France, 2007. MR 2780321.

[21] C. Series, "On coding geodesics with continued fractions" in Ergodic Theory (Sem., Les Plans-sur-Bex, 1980), Enseign. Math. (2) 29, Université de Genève, Geneva, 1981, 67-76. MR 0609896.

\author{
Alex Degtyarev \\ Department of Mathematics \\ Bilkent University \\ 06800 Ankara \\ Turkey \\ degt@fen.bilkent.edu.tr
}


Nermin Salepci

Institut Camille Jordan

69622 Villeurbanne CEDEX

France

salepci@math.univ-lyon1.fr 